Noname manuscript No.

(will be inserted by the editor)

\section{A robust composite time integration scheme for snap-through problems}

\author{
Yenny Chandra . \\ Yang Zhou • Ilinca \\ Stanciulescu - Thomas \\ Eason · Stephen \\ Spottswood
}

Received: date / Accepted: date

\begin{abstract}
A robust time integration scheme for snapthrough buckling of shallow arches is proposed. The algorithm is a composite method that consists of three sub-steps. Numerical damping is introduced to the system by employing an algorithm similar to the backward differentiation formulas (BDF) method in the last substep. Optimal algorithmic parameters are established based on stability criteria and minimization of numerical damping. The proposed method is accurate, numerically stable, and efficient as demonstrated through several examples involving loss of stability, large deformation, large displacements and large rotations.
\end{abstract}

\section{Introduction}

Dynamic snap-through buckling is a particular concern for many thin curved structures as it can exacerbate the fatigue failure $44,46,20,21$. In the context of fatigue life, being able to determine whether the system will continually snap between remote configurations or oscillate locally about a snapped configuration is important and constitutes the motivation for this work. However, the transient simulation of such highly nonlinear phenomena involving large deformations is very

Y. Chandra, Y. Zhou and I. Stanciulescu

Rice University, Department of Civil and Environmental Engineering, Houston, TX, 77005, U.S.A

Tel.: +1 7133484704

Fax: +1 17133485268

E-mail: ilinca.s@rice.edu

T. Eason and S. Spottswood

Air Force Research Laboratory, Structural Sciences Center, 2790 D. Street, WPAFB, OH, U.S.A. challenging, in many cases leading to inaccurate predictions [19, 17, 18. With the goal of predicting postsnapped behavior, the focus is on developing a robust time integration scheme with reliable numerical dissipation for spurious high order modes to resolve numerical issues encountered in the simulation of structures undergoing snap-through.

Classical time-stepping schemes that are unconditionally stable in the linear range can lead to unstable solutions for nonlinear problems. The numerical instability is often accompanied by an uncontrolled growth of energy. Such observation has motivated the development of energy conserving schemes. Following the pioneering work by Simo and Tarnow [40], many studies extended this conserving algorithm to general hyperelastic materials [24,33,35,16] and arbitrary geometric nonlinearities 38,39. Other work focused on applying these algorithms to specific finite element formulations (beam and shell elements) [41,42, 15, 48, and multi-body systems [7, 10, 28]. Despite the achievement of unconditional stability, the energy conserving schemes still show difficulties for numerically stiff nonlinear problems [7,1,2, and especially for snap-through buckling problems [31,32. The energy conserving schemes fail to converge in the post-snap range with an acceptable time step, due to the lack of numerical dissipation for spurious high-frequency oscillations. In the study of this class of problems, the analyst generally prefers time integration algorithms that are unconditionally stable with quantifiable numerical dissipation to suppress spurious high frequency components while minimizing the effects on accuracy.

Time stepping schemes with effective numerical dissipation were first developed for linear problems 34,25 , 47, 22. However, when used for nonlinear problems the characteristics of the numerical dissipation will change allowing the spurious high frequency oscillations to pollute the computation. Energy decaying schemes were proposed as a way to achieve more reliable numerical dissipation for nonlinear problems. Energy decaying schemes can be generally divided into two approaches. In the first approach, the time-discontinuous Galerkin formulation was utilized to predict the behavior of nonlinear beams [8,12,13] and multi-body systems [9,10, 11,14. These algorithms involve a multistage computation per time step and require solving a larger system than those solved with classical schemes, due to the coupling between the displacement and velocity. Another approach is to construct the algorithm from an energy conserving integrator $32,30,1,2,36,27]$. These algorithms are designed using a specialized approximation of the element stress tensor and the velocity to ensure a positive numerical damping. Like many energy 
conserving schemes, an energy decaying method cannot be generalized across different finite element formulations (e.g., different shell elements). Moreover, the resultant tangent stiffness matrix is usually non-symmetric, leading to increased computational costs.

An alternate method to construct time integration methods with reliable numerical dissipation for nonlinear problems is the use of a composite algorithm as presented by Bathe and coworkers [5, 3, 4, 6]. The main idea of this approach is to combine a non-dissipative method and a dissipative method into a one-step but multistage composite scheme. Bathe and coworkers combined the trapezoidal rule and the three-point backward Euler formula into a one step two-stage composite scheme. Although the composite algorithm requires roughly twice the computational cost as the trapezoidal rule per time step, much larger time steps can be used for large deformations nonlinear dynamics. Following a similar line of thought, Dong [23] proposed two generalized backward differentiation formulas to build a composite time integrator consisting of the trapezoidal rule and a generalized backward differentiation formula.

Motivated by introducing reliable numerical damping in simulating snap-through problems, and in particular to obtain accurate post-snap predictions, the ideas from Bathe [5, 3, 4,6] and Dong [23] were extended to develop a new composite time-stepping scheme with $\mathrm{im}$ proved numerical dissipation characteristics. The new composite algorithm consists of three sub-steps where the trapezoidal rule is used to perform the first and second sub-steps, while a GBDF method [23], is employed in the third sub-step to introduce numerical dissipation. This new method is self-starting and does not require a special modification of element stress tensor. Its implementation is quite straightforward, remains the same for different finite element formulations, and preserves the symmetry of the tangent stiffness matrix. The proposed scheme has two algorithmic parameters to control the numerical dissipation and their optimized values to minimize numerical damping are determined. For the rest of this paper, the new composite scheme will be referred to as the TTBDF (Trapezoidal-TrapezoidalBackward-Difference-Formula) method.

The improved numerical dissipation of the TTBDF method over other composite schemes from Bathe and Dong is first demonstrated analytically by the comparison of spectral radii, algorithmic damping ratios, and relative period elongations. Although the stability and dissipation analysis is limited to the linear range, numerical simulations of curved beams undergoing snapthrough confirm that the TTBDF method has better numerical dissipation properties than other existing composite schemes. The TTBDF method leads to more ac- curate solutions for long-time record simulations using amplitude decay and period elongation as a metric. Further, the total cost of the computation can be reduced using the TTBDF method through the use of larger time steps for the same accuracy as compared to other composite methods. It is also shown that the TTBDF method has better numerical dissipation when applied to other nonlinear dynamic problems having large deformations and rotations. The examples include a rigid and a deformable pendulum, a free moving cylindrical shell and a three dimensional L-shaped block, which were used as benchmark problems in [23, 40, 30, 4, 7, 37. to evaluate the time integrators proposed in the respective papers.

This paper is organized as follows. In Section 2, the proposed numerical scheme is described, including the approach to determine optimal values for the algorithmic parameters. Section 3 demonstrates the robustness of the proposed method through several examples. Concluding remarks are presented in the last section.

\section{TTBDF time integration scheme}

Consider the nonlinear undamped equations of structural dynamics

$\mathbf{M u}+\mathbf{N}(\mathbf{u}, t)=\mathbf{R}(t)$

with appropriate initial conditions. $\mathbf{M}$ is the mass matrix, $\mathbf{N}$ is the vector of internal forces, $\mathbf{R}$ is the vector of external forces, $\mathbf{u}$ is the displacement vector, $t$ is time, and superposed dot indicates differentiation with respect to time. Approximate solutions of Eq. 1 at discrete time points are obtained using the time discretized equation given by

$\mathbf{M} \ddot{\mathbf{u}}_{n}+\mathbf{N}_{n}=\mathbf{R}_{n}$

where $n$ is the time step index.

A three-stage composite time-stepping scheme is used to solve Eq. 2 using the trapezoidal rule for the first and second sub-steps and a BDF-like algorithm in the third sub-step . Let $\Delta t$ be the time step size; the time step for each sub-step is $\delta t=\frac{\Delta t}{3}$. In the first and second sub-steps of the $(n+1)$-th time step, the discretized equation is enforced at $n+\frac{1}{3}$ and $n+\frac{2}{3}$. Thus, the updates for the first sub-step $\left(n+\frac{1}{3}\right)$ are given by

$\mathbf{u}_{n+\frac{1}{3}}=\mathbf{u}_{n}+\frac{\Delta t}{6}\left(\dot{\mathbf{u}}_{n}+\dot{\mathbf{u}}_{n+\frac{1}{3}}\right)$

$\dot{\mathbf{u}}_{n+\frac{1}{3}}=\dot{\mathbf{u}}_{n}+\frac{\Delta t}{6}\left(\ddot{\mathbf{u}}_{n}+\ddot{\mathbf{u}}_{n+\frac{1}{3}}\right)$

Similar with the first sub-step, by applying the the trapezoidal rule, the updates for the second sub-step $\left(n+\frac{2}{3}\right)$ are as follows

$\mathbf{u}_{n+\frac{2}{3}}=\mathbf{u}_{n+\frac{1}{3}}+\frac{\Delta t}{6}\left(\dot{\mathbf{u}}_{n+\frac{1}{3}}+\dot{\mathbf{u}}_{n+\frac{2}{3}}\right)$

$\dot{\mathbf{u}}_{n+\frac{2}{3}}=\dot{\mathbf{u}}_{n+\frac{1}{3}}+\frac{\Delta t}{6}\left(\ddot{\mathbf{u}}_{n+\frac{1}{3}}+\ddot{\mathbf{u}}_{n+\frac{2}{3}}\right)$ 
In the third sub-step, the GBDF-A algorithm proposed by Dong is employed [23] and the updates for the GBDFA algorithm are as follows

$$
\begin{aligned}
\dot{\mathbf{u}}_{n+1}=\frac{A\left(\theta_{1}\right)}{\Delta t / 3} \mathbf{u}_{n+1}+\frac{B\left(\theta_{1}\right)}{\Delta t / 3} \mathbf{u}_{n+\frac{2}{3}}+ & \frac{C\left(\theta_{1}\right)}{\Delta t / 3} \mathbf{u}_{n+\frac{1}{3}} \\
& +\frac{D\left(\theta_{1}\right)}{\Delta t / 3} \mathbf{u}_{n} \\
\ddot{\mathbf{u}}_{n+1}=\frac{A\left(\theta_{2}\right)}{\Delta t / 3} \dot{\mathbf{u}}_{n+1}+\frac{B\left(\theta_{2}\right)}{\Delta t / 3} \dot{\mathbf{u}}_{n+\frac{2}{3}}+ & \frac{C\left(\theta_{2}\right)}{\Delta t / 3} \dot{\mathbf{u}}_{n+\frac{1}{3}} \\
& +\frac{D\left(\theta_{2}\right)}{\Delta t / 3} \dot{\mathbf{u}}_{n}
\end{aligned}
$$

where

$$
\begin{aligned}
& A(\theta)=\frac{11}{6}-\frac{\theta}{3} \\
& B(\theta)=\theta-3 \\
& C(\theta)=\frac{3}{2}-\theta \\
& D(\theta)=-\frac{1}{3}+\frac{\theta}{3}
\end{aligned}
$$

For analysis and comparison purposes, linear stability analysis is used and the orthogonality property is invoked to reduce the coupled equations of motion and the algorithmic equations to a series of uncoupled single-degree-of freedom systems [26]. The singledegree-of-freedom problem is given by

$\ddot{u}+\omega^{2} u=0$

and initial conditions $u(0)=1, \dot{u}(0)=0$, where $u$ is the unknown variable and $\omega$ is the angular frequency of oscillation. Eq. (7) is discretized by employing the TTBDF scheme resulting in an iterative relation as follows.

$$
\left[u^{n+1}, \dot{u}^{n+1}\right]^{T}=\mathbf{G}\left[u^{n}, \dot{u}^{n}\right]^{T},
$$

where $\mathbf{G}$, the amplification matrix, is given by

$$
\mathbf{G}=\left[\begin{array}{cc}
G_{11} & G_{12} \Delta t \\
G_{21} \frac{1}{\Delta t} & G_{22}
\end{array}\right]
$$

with

$$
\begin{gathered}
G_{11}=\frac{1}{M}\left(2 \theta_{1}\left(-11+2 \theta_{2}\right)\left(1296-576(\omega \Delta t)^{2}+7(\omega \Delta t)^{4}\right)\right. \\
+156816-64512(\omega \Delta t)^{2}+1399(\omega \Delta t)^{4} \\
-2 \theta_{2}\left(14256-6336(\omega \Delta t)^{2}+245(\omega \Delta t)^{4}\right)
\end{gathered}
$$

$$
\begin{aligned}
G_{12}= & \frac{2}{M}\left(78408-6714(\omega \Delta t)^{2}+29(\omega \Delta t)^{4}\right. \\
& -108 \theta_{1}\left(-11+2 \theta_{2}\right)\left(-12+(\omega \Delta t)^{2}\right) \\
& \left.-2 \theta_{2}\left(7128-846(\omega \Delta t)^{2}+7(\omega \Delta t)^{4}\right)\right)
\end{aligned}
$$

$$
\begin{aligned}
G_{21}= & \frac{2(\omega \Delta t)^{2}}{M}\left(-78408+6714(\omega \Delta t)^{2}-29(\omega \Delta t)^{4}\right. \\
& +\left(216 \theta_{1} \theta_{2}-1188 \theta_{2}\right)\left(-12+(\omega \Delta t)^{2}\right) \\
& \left.+2 \theta_{1}\left(7128-846(\omega \Delta t)^{2}+7(\omega \Delta t)^{4}\right)\right)
\end{aligned}
$$

$$
\begin{gathered}
G_{22}=\frac{1}{M}\left(156816-64512(\omega \Delta t)^{2}+1399(\omega \Delta t)^{4}\right. \\
+\left(4 \theta_{1} \theta_{2}-22 \theta_{2}\right)\left(1296-576(\omega \Delta t)^{2}+7(\omega \Delta t)^{4}\right) \\
\left.+2 \theta_{1}\left(-14256+6336(\omega \Delta t)^{2}-245(\omega \Delta t)^{4}\right)\right) \\
M=\left(36+(\omega \Delta t)^{2}\right)^{2}\left(121-22 \theta_{2}+\theta_{1}\left(-22+4 \theta_{2}\right)\right. \\
\left.+4(\omega \Delta t)^{2}\right) .
\end{gathered}
$$

Let $\rho_{G}\left(\theta_{1}, \theta_{2}, \omega \Delta t\right)$ be the spectral radius of the amplification matrix for a specific $\omega \Delta t$. The maximum spectral radius is defined as

$\rho \max \left(\theta_{1}, \theta_{2}\right)=\max _{0 \leqslant \omega \Delta t \leqslant \infty} \rho_{G}\left(\theta_{1}, \theta_{2}, \omega \Delta t\right)$.

An algorithm is unconditionally stable for linear problems if $\rho_{\max } \leqslant 1$ for any time step size $0 \leqslant \frac{\Delta t}{T} \leqslant \infty$, where $T$ is the period of oscillation, $T=\frac{2 \pi}{\omega}$. The unshaded region in Fig. 1a shows the region where $\rho_{\max } \leqslant$ 1.

Next the effect of $\theta_{1}$ and $\theta_{2}$ on the dissipativity of the TTBDF algorithm is investigated by evaluating the energy loss over time. The total energy of the linear vibration is defined by $E_{t}=\frac{1}{2} \omega^{2} u^{2}+\frac{1}{2} \dot{u}^{2}$. If the initial energy is denoted by $E_{0}$, the decay over time is defined by the function, $E_{t}=E_{0} e^{-\gamma t / T}$. The constant $\gamma$ depends on $\theta_{1}, \theta_{2}$ and $\Delta t / T$. The fraction of energy loss per period $\frac{E_{0}-\left.E(t)\right|_{t=T}}{E_{0}}=1-e^{-\gamma}$ is then used as a measure of the dissipativity of the algorithm. The contour plot of the fraction of energy loss shows that the algorithm becomes more dissipative as $\left(\theta_{1}, \theta_{2}\right)$ increase in the domain of unconditional stability (Fig. 1b). In Section 3 . the simulations are performed with $\theta_{1}=\theta_{2}=0.75$; these values are among the least dissipative of this class of schemes and therefore, they are preferred, as shown in Fig. 2a. The fraction of energy loss as a function of $\frac{\Delta t}{T}$ for this case is shown in Fig. 2b. As expected, the amount of dissipation increases as $\Delta t$ increases. It is expected that the algorithm will show increased damping with increased time step in nonlinear problems.

Finally, the properties of the proposed algorithm are compared with those of other schemes from the BDF class and with composite schemes that have a member of BDF class as a sub-step. Specifically Bathe's method [5, 3, 4, 6 and the algorithms proposed by Dong [23, the GBDF-A, GBDF-B, and GBDF-TR methods are chosen for comparison since they possess numerical dissipation while maintaining accuracy. Bathe's algorithm is a two-stage composite method with trapezoidal and backward-difference used as sub-steps. The GBDF-A is a four-point BDF-like scheme and the GBDF-B is a five point BDF-like method. The GBDF-TR is a composite two-stage algorithm that consists of sub-steps using the trapezoidal rule and the GBDF-B method. Bathe's method has no algorithmic parameters. The GBDF-A, GBDF-B, and GBDF-TR have two algorithmic parameters each; their values (as recommended by Dong [23]) are listed in Table 1 . These parameters lead to minimal damping in linear vibration analysis and to unconditional stability based on linear stability analysis.

The algorithmic properties compared are quantities used as measures of the accuracy: the spectral radius, the algorithmic damping ratio, and the relative 


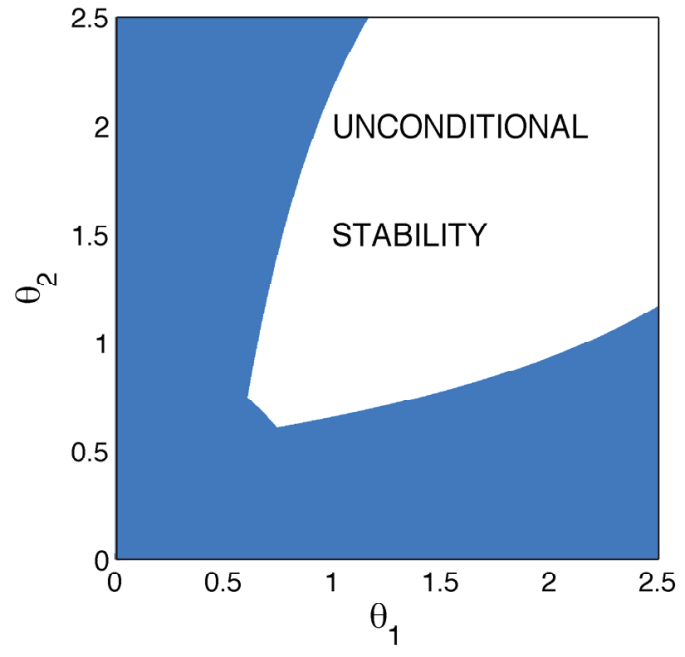

(a)

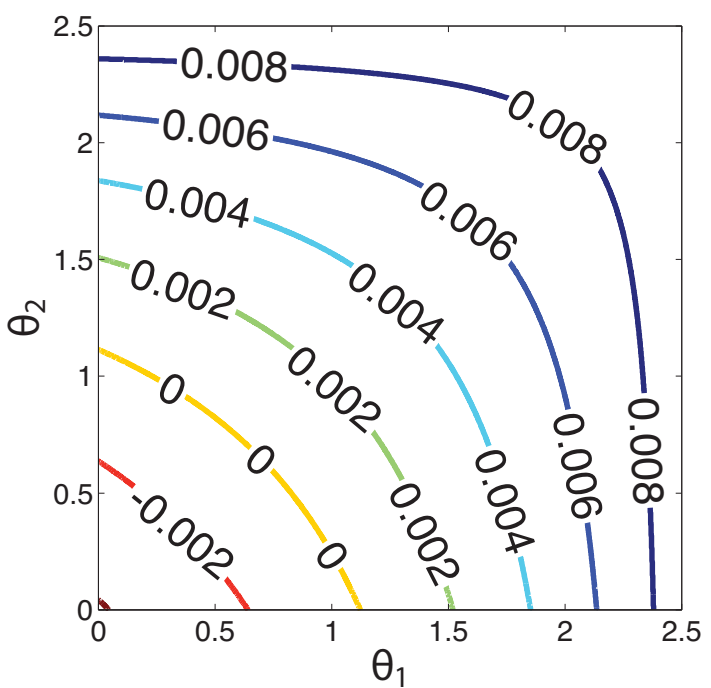

(b)

Fig. 1: TTBDF algorithm: (a) region of unconditional stability and (b) contours of fraction energy loss per period at $\frac{\Delta t}{T}=0.1$.

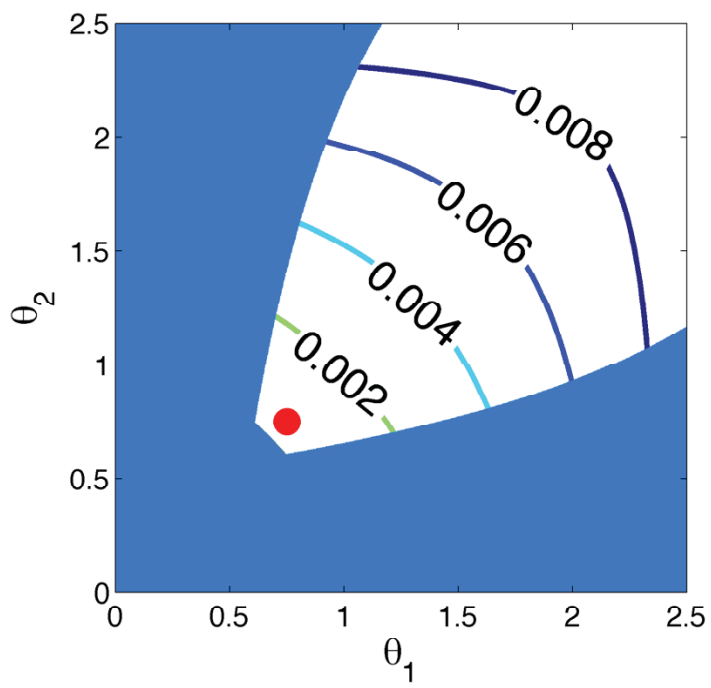

(a)

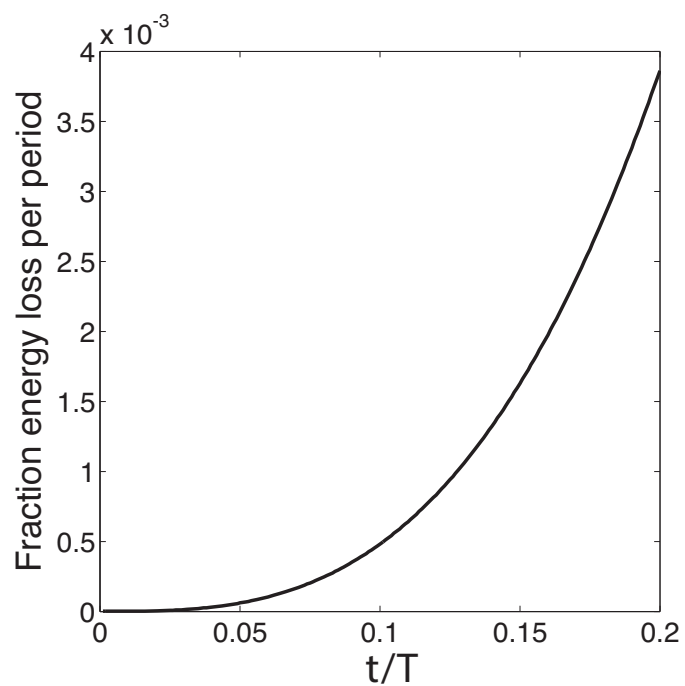

(b)

Fig. 2: TTBDF algorithm: (a) area of unconditional stability superposed with contours of energy loss with $\theta_{1}=\theta_{2}=3 / 4$ (red dot) used in the simulations, (b) fraction of energy loss for $\theta_{1}=\theta_{2}=3 / 4$.

Table 1: Algorithmic Parameters

\begin{tabular}{|l|c|c|}
\hline Algorithms & $\theta_{1}$ & $\theta_{2}$ \\
\hline GBDF-A & 1 & 0 \\
GBDF-B & $1 / 2$ & $4 / 5$ \\
GBDF-TR & $7 / 9$ & $9 / 10$ \\
TTBDF & $3 / 4$ & $3 / 4$ \\
\hline
\end{tabular}

period error. The spectral radius is an important measure of numerical dissipation. It is often necessary to have numerical dissipation in order to eliminate the high-frequency behavior due to the discretization process while maintaining good accuracy in the low frequency region. Therefore, a preferred spectral radius has a value close to unity in the low frequency domain, but approaching zero in the high-frequency range. The proposed method (TTBDF) has a better spectral radius than other algorithms since its spectral radius maintains closer to one for larger range of $\Delta t / T$ when $\Delta t / T$ 
is small, and goes to zero when $\Delta t / T$ is large, as shown in Fig. 3a. The improved performance can be seen more clearly when comparing the algorithmic damping ratio and relative period error. The algorithmic damping ratio is a measure of the numerical dissipation, while the relative period error is a measure of the numerical dispersion. According to [25,22], one pair of complex conjugate eigenvalues $\lambda_{a, b}$ of the amplification matrix can be expressed as

$\lambda_{a, b}=\exp [\bar{\omega} \Delta t(-\bar{\xi} \pm i)]$

$\bar{\xi}$ is the algorithmic damping ratio and $(\bar{T}-T) / \bar{T}$ with $\bar{T}=2 \pi / \bar{\omega}$ the relative period error. The algorithmic damping ratios and the relative period errors of the algorithms investigated are shown in Figs. $3 \mathrm{~b}$ and $3 \mathrm{c}$. respectively. The TTBDF method has less numerical dissipation and smaller period error in low-frequency range compared to the other algorithms.

The primary motivation for developing the TTBDF integrator came from the relatively poor performance in snap-through analyses of methods that were otherwise performing very well on standard problems. For such systems whose (physical) response can be unstable, one should not consider unconditional stability as the sole measure of algorithmic stability performance. Instead, consistent stability should be the goal. It is possible that the real response is divergent and yet the integrator produces a stable oscillation (shown in [29]). From an engineering perspective, this case, that was so far ignored, leads to an under-conservative prediction of the response. Even though it leads to retrieving the correct stability in the case of stable systems, unconditional stability does not necessarily imply that the prediction is a good approximation of the true solution, but only that it remains bounded. Unconditionally stable integrators may, when applied to complex systems with multiple equilibria, predict trajectories that are non-physical and/or greatly underestimate the severity of the response [45. Following similar methods with those used in 29,45, a bound on the time step size can be determined to enforce the condition that the algorithm will not predict a stable response for an unstable linear system. The maximal time steps for the methods compared in this paper are shown in Fig. $3 \mathrm{~d}$ and demonstrate the good performance of TTBDF for this criterion as well.

Since the TTBDF method is a three-stage method while the other methods compared are either a onestage (the GBDF-A and the GBDF-B methods) or twostage (the GBDF-TR and Bathe's methods) methods, each time step of the TTBDF method is more expensive. However, often times a larger time step can be used with the TTBDF method as can be seen in the snap-through example given in Section 3.1 and the free moving shell example in Section 3.3 .

\section{Representative numerical examples}

Systems undergoing snap-through are the main motivation of the development of this algorithm and are the first examples in this section that demonstrate the robustness of the TTBDF method. In addition, several other examples are presented to evaluate the performance of the proposed time integration scheme in solving other numerically challenging problems involving large deformation, large displacements, and large rotations. The numerical simulations are performed with Matlab and the Finite Element Analysis Program (FEAP), a research code developed at UC Berkeley. FEAP includes most commonly used finite elements and solvers and provides a reliable framework for developing and implementing new user formulations [43].

\subsection{Curved arches}

Circular arches with clamped supports are investigated to demonstrate the capability of the proposed integrator in simulating systems undergoing snap-through. The arch is a thin curved beam, symmetrical, with an angle $\theta$ at the supports, and horizontal projection $L$ (Fig. 4). The cross section is rectangular with depth $d$ much larger than the thickness $t$.

Table 2: Geometry of Curved Beams

\begin{tabular}{|c|c|c|c|c|}
\hline Beam & $\begin{array}{c}\text { Radius R } \\
{[\mathrm{mm} .]}\end{array}$ & $\begin{array}{c}\text { Initial Rise M } \\
{[\mathrm{mm}]}\end{array}$ & $\begin{array}{c}\text { Curvature } \kappa \\
{[1 / \mathrm{mm}]}\end{array}$ & $\begin{array}{c}\theta \\
{[\mathrm{rad}]}\end{array}$ \\
\hline 1 & 762 & 15.39553 & $1.312 \mathrm{e}-3$ & 0.2014 \\
2 & 3048 & 3.8124 & $0.328 \mathrm{e}-3$ & 0.0500 \\
\hline
\end{tabular}

Table 3: Material properties

\begin{tabular}{|l|r|}
\hline Properties & Values \\
\hline Young's Modulus $\left[\mathrm{N} / \mathrm{mm}^{2}\right]$ & 206843 \\
Poisson's Ratio & 0.28 \\
Density $\left[\mathrm{N} \mathrm{s}^{2} / \mathrm{mm}^{4}\right]$ & $7.83 \times 10^{-9}$ \\
\hline
\end{tabular}

Two angles $\theta$ are considered; the geometry of the two beams is described in Table 2 where $M$ is the initial height of the arch at the midpoint, $\kappa=1 / R$ is the curvature of the beam, and $\theta$ is the angle at the supports. The projection length is $L=12 \mathrm{in}$. (304.8 mm) for all beams, and the thickness is $t=0.024 \mathrm{in}$. (0.6096 
$\mathrm{mm})$. A transverse depth $d=0.5 \mathrm{in} .(12.7 \mathrm{~mm})$ is used. Material properties are those of steel, given in Table 3 . The comparison of the beams' curvatures is shown in Fig. 5 .

For discretization, 2D straight beam elements are used. The beam elements utilized are beam elements without shear deformation with large displacement and small rotation ( $2^{\text {nd }}$ order theory) with cubic interpolation 43. A study on the performance of other elements (2D beam element with shear deformation, 3D linear displacement formulation, B-bar, and enhanced formulations, and 3D quadratic element) is presented in [19. 17.

The load is a ramp load applied at the midpoint of the arch. It initially increases to some value above the snap-through limit, with a loading rate of $1 \mathrm{~N} / \mathrm{s}$ and then is kept constant for a certain time. The applied load is as follows:

For beam1, $F= \begin{cases}t & 0 \leqslant t \leqslant 8 \\ 8 & 8<t\end{cases}$

For beam2, $F= \begin{cases}t & 0 \leqslant t \leqslant 2 \\ 2 & 2<t\end{cases}$

Fig. 6 shows the solutions of Beams 1 and 2 obtained with $\Delta t=10^{-3} \mathrm{~s}$ and $\Delta t=2 \times 10^{-4} \mathrm{~s}$, respectively. These time steps are chosen such that the true solution is obtained [19,17, 18]. The figure shows that the systems oscillate about their snapped configuration starting immediately after the snap-through event; the amplitude of the oscillations decreases over the time interval where the load continues to ramp up, but then remains constant when the external load is constant.

The deformed configurations of Beams 1 and 2 obtained by the TTBDF algorithm at several different time instances are shown in Figs. $7 \mathrm{a}$ and $7 \mathrm{~b}$ respectively. It can be clearly observed that both beams display severe curvature reversals when experiencing dynamic snap-through buckling.

The variations of total energy of Beam 1 obtained using the GBDF-A, GBDF-B, GBDF-TR, Bathe's method and the TTBDF, are presented in Fig. 8 . Fig. 8a shows the total energy when $\Delta t=10^{-3} \mathrm{~s}$ is used. For all algorithms, the energy decreases when the arch snapsthrough; the TTBDF method gives the smallest dissipation. The total energy for the TTBDF method decreases slightly at the snap-through point and then stays constant in the region where the external load remains constant. The GBDF-A, GBDF-B, GBDF-TR and Bathe's method result in a similar amount of decrease in total energy due to the numerical damping although the GBDF-TR damps at a slower rate in the beginning.
The amount of damping in these four methods is much larger than that of the TTBDF method.

The TTBDF method consists of three sub-steps, while the other methods only have one (the GBDF-A and GBDF-B methods) or two sub-steps (the GBDFTR and Bathe's methods). Thus, it might be argued that the TTBDF method results in the smallest dissipation due to a smaller time step size of each sub-step. Therefore, the performance of these integrators is compared for equal sub-step size (same total number of system solves or matrix inversions). Each sub-step in the TTBDF method is performed with $\delta t=\Delta t / 3=1 / 3 \times 10^{-3} \mathrm{~s}$. To ensure a fair comparison, the GBDF-A and GBDF-B methods (one sub-step), will use $\Delta t=\delta t$. The GBDFTR and Bathe's methods (two sub-steps), are performed with $\Delta t=2 \delta t=2 / 3 \times 10^{-3} \mathrm{~s}$. The total energy of these algorithms is shown in Fig. $8 \mathrm{~b}$. The figure indicates a slower rate in the decrease of energy for one sub-step and two sub-steps methods than that shown in Fig. $8 \mathrm{a}$. Bathe's method results in the fastest and largest decrease of energy, followed by the GBDF-A, GBDF-TR, GBDF-B, and the TTBDF method.

The amplitude decay of the response presented in Fig. 9 show the effect of the algorithmic damping for each algorithm. The response obtained using the TTBDF method is constant during the period of constant load while responses obtained with other algorithms show a significant amount of damping. It can be concluded that the proposed method has the best performance compared with other algorithms for this problem as it has the smallest dissipation, therefore the accuracy is not compromised.

Similar analysis is performed for Beam 2, a shallower arch than Beam 1. In Fig. 10a, the total energy obtained with $\Delta t=2 \times 10^{-4} \mathrm{~s}$ for various algorithms is presented. For this beam, the GBDF-A shows the largest dissipation initially, followed by the GBDF-B method. Bathe's method shows a large jump in dissipation at around $t=18 \mathrm{~s}$. The GBDF-A and the GBDF-B have similar dissipation right after snap-through, however, the energy of the GBDF-A method decreases at a faster rate. The TTBDF and the GBDF-TR methods have the best performance.

Fig. $10 \mathrm{~b}$ compares the total energy when each substep of all the methods is performed with $\delta t=2 / 3 \times$ $10^{-4} \mathrm{~s}$ for a fair comparison of computational effort. Bathe's method shows the largest amount of dissipation, while the GBDF-B, GBDF-TR, GBDF-A and TTBDF methods are fairly similar in performance. Overall, the dissipation is quite small for this shallower arch.

The dissipation can be seen in the amplitude decay of the response presented in Fig. 11. For $\Delta t=2 \times 10^{-4} \mathrm{~s}$, the responses obtained using the TTBDF method and 
the GBDF-TR method show the least amount of amplitude decay. For simulations with $\delta t=2 / 3 \times 10^{-4}$, all algorithms except Bathe's method have a very small dissipation.

This example shows that the proposed method is capable of simulating arches undergoing snap-through. The simulations are stable and for taller arches, this method results in smaller dissipation than all the other methods investigated here. This advantage is not only shown when the same time step size in each step $\Delta t$ is used but also when the same time step size for each sub-step $\delta t$ is used. For shallower arches, the differences between methods are less significant. Therefore, it can be concluded that the TTBDF algorithm is robust and introduces minimal dissipation in the simulations of curved beams in general and is the most efficient among the algorithms examined in the case of taller arches.

\subsection{Pendulums}

Numerical examples of a rigid and a deformable pendulum are considered here to demonstrate the capability of the TTBDF method in the transient analysis of 2D nonlinear dynamic systems with large deformations. These two examples were considered in 4 to show the effectiveness of Bathe's composite method.

\subsubsection{Rigid pendulum}

A vertical pendulum pined at the upper end, with axial stiffness $\mathrm{EA}_{0}=10^{10} \mathrm{~N}$, density $\rho \mathrm{A}_{0}=6.57 \mathrm{~kg} / \mathrm{m}$, and length $\mathrm{l}_{0}=3.0443 \mathrm{~m}$, is subjected to an initial horizontal velocity $\left(\mathrm{v}_{0}=7.72 \mathrm{~m} / \mathrm{s}\right)$ and radial acceleration $\left(\mathrm{a}_{0}=19.6 \mathrm{~m} / \mathrm{s}^{2}\right)$ at its free end. The pendulum displays an almost rigid-body rotation due to the large axial stiffness.

The performance of the TTBDF and Bathe's method is evaluated by comparing the amplitude and period errors of the vertical displacement and velocity for four different time steps $(\Delta t=0.05 \mathrm{~s}, 0.1 \mathrm{~s}, 0.2 \mathrm{~s}$, and 0.4 s) at $t=50 \mathrm{~s}$. Solutions obtained with a very small time step $\Delta t=0.01 \mathrm{~s}$ are used as reference values to calculate these errors.

The amplitude errors of the vertical displacement, resulting from using these two methods are very small, less than $0.6 \%$ for the largest time step (Fig. 12a). However, Bathe's method produces much larger period errors than the TTBDF method (Fig 12b). The period errors of Bathe's method become unreasonably large ( $47.15 \%$ for $\Delta t=0.4 \mathrm{~s})$ at large time steps, while the errors of the TTBDF method are still very small (less than $1.5 \%$ for $\Delta t=0.4 \mathrm{~s})$. An obvious period elongation for Bathe's method over the TTBDF method at $\Delta t=0.4 \mathrm{~s}$ can be clearly observed in Fig. $13 \mathrm{a}$.

The amplitude errors of Bathe's method in evaluating the vertical velocity become much larger than those of the TTBDF method (Fig. 14a). For large time steps, the amplitude errors of Bathe's method are unacceptably large $(29.93 \%$ for $\Delta t=0.4 \mathrm{~s})$, while the TTBDF method still results in very small amplitude errors (less than $0.36 \%$ for $\Delta t=0.4 \mathrm{~s})$. A significant amplitude decay for Bathe's method over the TTBDF method can be identified at $\Delta t=0.4 \mathrm{~s}$ in Fig. $13 \mathrm{~b}$. The period errors of the velocity (Fig $14 \mathrm{~b}$ ) are similar to those errors of the displacement (Fig 12b).

\subsubsection{Elastic pendulum}

The elastic pendulum has the same initial length and density as the rigid one, but different axial stiffness $\left(\mathrm{EA}_{0}=10^{4} \mathrm{~N}\right)$. The initial horizontal velocity of the elastic beam is $\mathrm{v}_{0}=7.72 \mathrm{~m} / \mathrm{s}$ and its initial vertical acceleration is $a_{0}=0 \mathrm{~m} / \mathrm{s}^{2}$.

The displacement and velocity amplitude and period errors of both methods for the elastic pendulum (Figs. 15 and 16) have similar patterns to those observed in the analysis of the rigid pendulum (Figs. 12 and 14.

The examples on rigid and elastic pendulums show that the TTBDF method produces stable and accurate solutions in simulating $2 \mathrm{D}$ nonlinear dynamic systems with either large rigid-body rotations or elastic displacements. Although Bathe's method leads to stable solutions, it introduces too much numerical damping at large time steps leading to inaccurate results.

\subsection{Structures with large 3D rotations}

To evaluate the performance of the TTBDF method in simulating nonlinear dynamic systems with large 3D rotations, a free moving cylindrical panel, and a rigid and a deformable 3D L-shaped blocks are investigated in this section. The free moving panel was utilized by Sansour et al. 37] to illustrate the performance of the energy-momentum time integrator they proposed and the L-shaped blocks were adopted by Dong [23] to demonstrate the capabilities of the GBDF-A, GBDF-B, and GBDF-TR algorithms.

\subsubsection{Free moving cylindrical panel}

A cylindrical panel subjected to two equal forces that are in positive y and $\mathrm{z}$ directions is considered (Fig. 17). 
The applied force $F(t)$ varies as follow:

$F(t)= \begin{cases}2 \times 10^{5} t & 0 \leqslant t<0.005 \\ 2000-2 \times 10^{5} t & 0.005 \leqslant t<0.01 \\ 0 & 0.01 \leqslant t\end{cases}$

Fig 18 shows the total energy of the panel for $0 \leqslant$ $t \leqslant 0.1$. The total energy of the system conserves well after the external force becomes zero. The solid line represents the solution calculated by the TTBDF algorithm with $\Delta t=2 \times 10^{-4}$, while the cross markers denote the results calculated by the energy-momentum method in 37] but with a much smaller time step $\Delta t=$ $1 \times 10^{-5}$. Although the TTBDF has three sub-steps in one time step, the time step that can be used by the TTBDF scheme is 20 times larger than the one for the energy-momentum method in [37] leading to a lower total computational cost of the TTBDF algorithm.

The snapshots of the panel configurations obtained by the TTBDF scheme at six different time sequences are shown in Fig. 19. This panel displays large overall 3D translations and rotations; the TTBDF method performs very well for this problem.

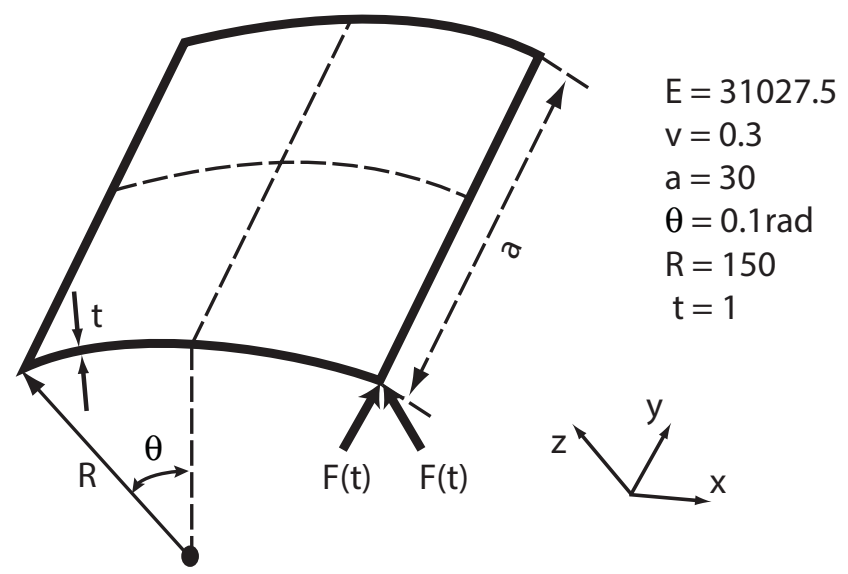

Fig. 17: Geometry and material properties of the cylindrical panel

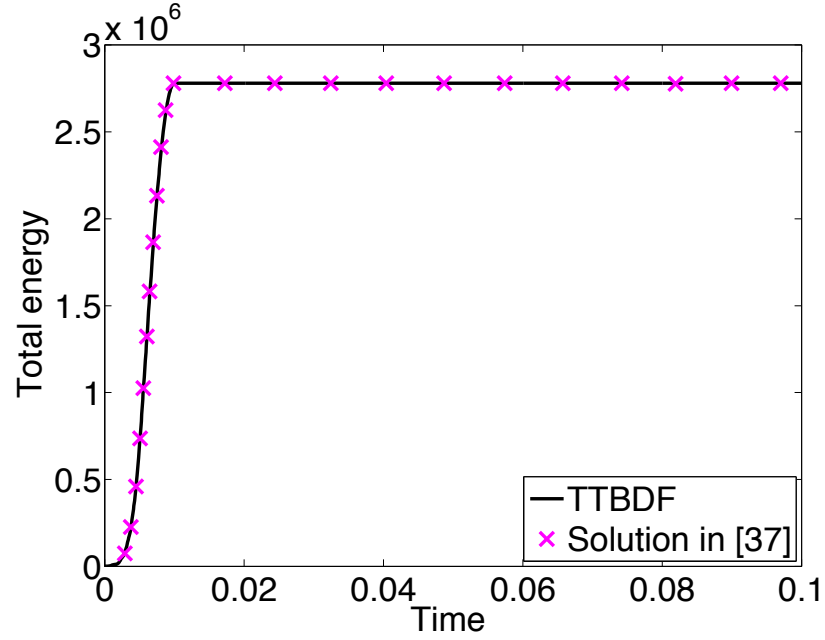

Fig. 18: Total energy of the cylindrical panel 


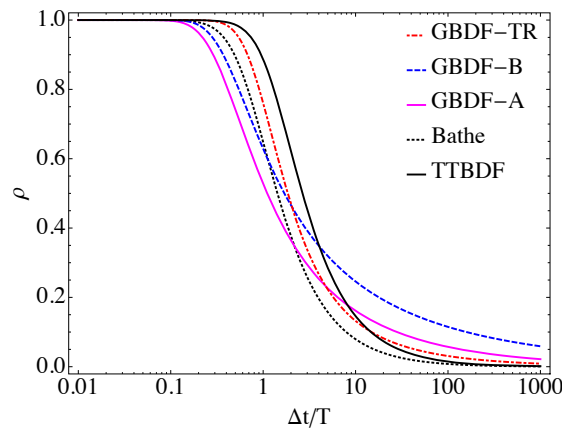

(a) Spectral radii

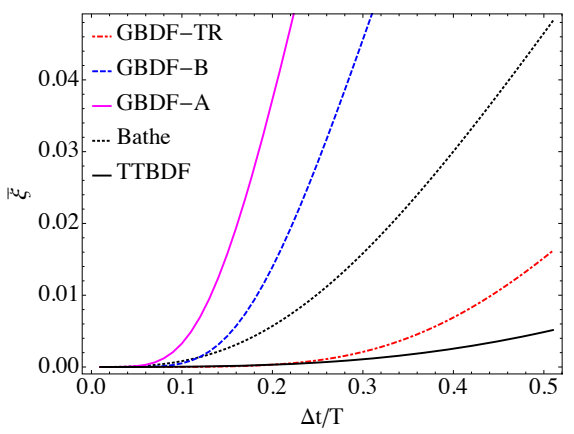

(b) Algorithmic damping ratios

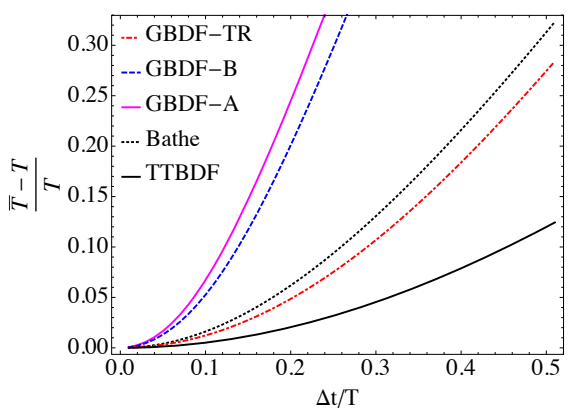

(c) Relative period errors

\begin{tabular}{|c|c|}
\hline TTBDF & $\frac{25.95}{\sqrt{-\omega^{2}}}$ \\
\hline BATHE & $\frac{12}{\sqrt{-\omega^{2}}}$ \\
\hline GBDF-A & $\frac{5.6}{\sqrt{-\omega^{2}}}$ \\
\hline GBDF-B & $\frac{6.5}{\sqrt{-\omega^{2}}}$ \\
\hline GBDF-TR & $\frac{4}{\sqrt{-\omega^{2}}}$ \\
\hline
\end{tabular}

(d) Largest time step for consistent stability

Fig. 3: Comparison of: (a) spectral radii, (b) algorithmic damping ratios, (c) relative period errors of numerically dissipative algorithms, and (d) step time bound for consistent stability. 

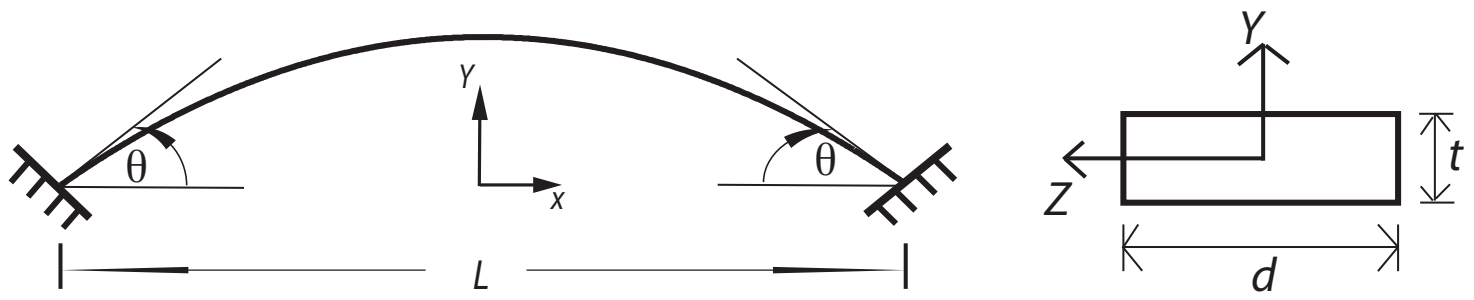

Fig. 4: Geometry of a curved beam clamped at the supports.

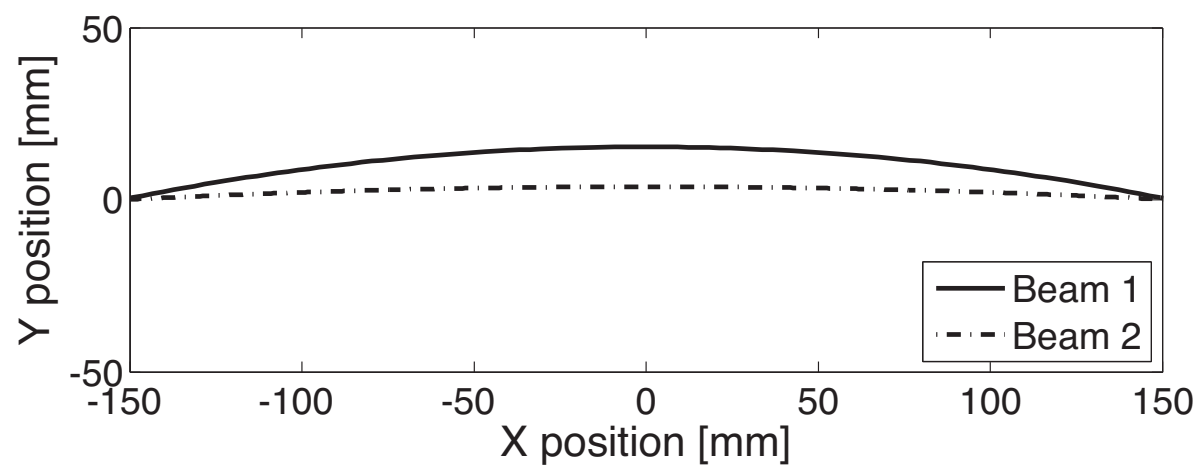

Fig. 5: Initial configurations of the two beams.

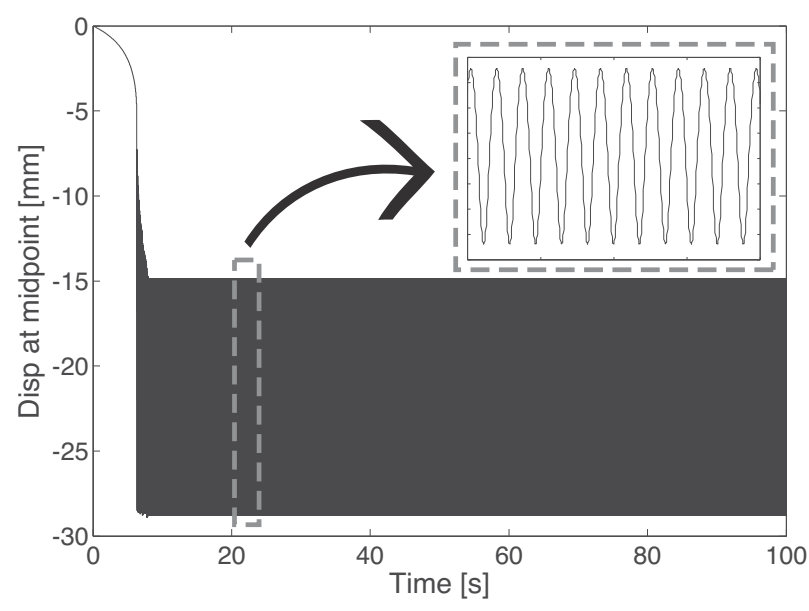

(a) Beam 1, $\Delta t=10^{-3} \mathrm{~s}$

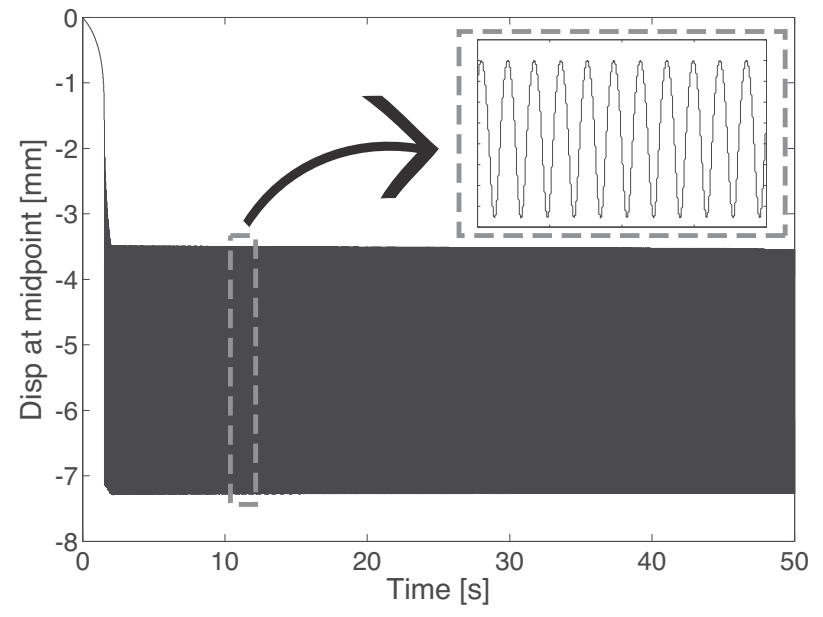

(b) Beam 2, $\Delta t=2 \times 10^{-4} \mathrm{~s}$

Fig. 6: Solutions of Beams 1 and 2 obtained using the TTBDF scheme. 


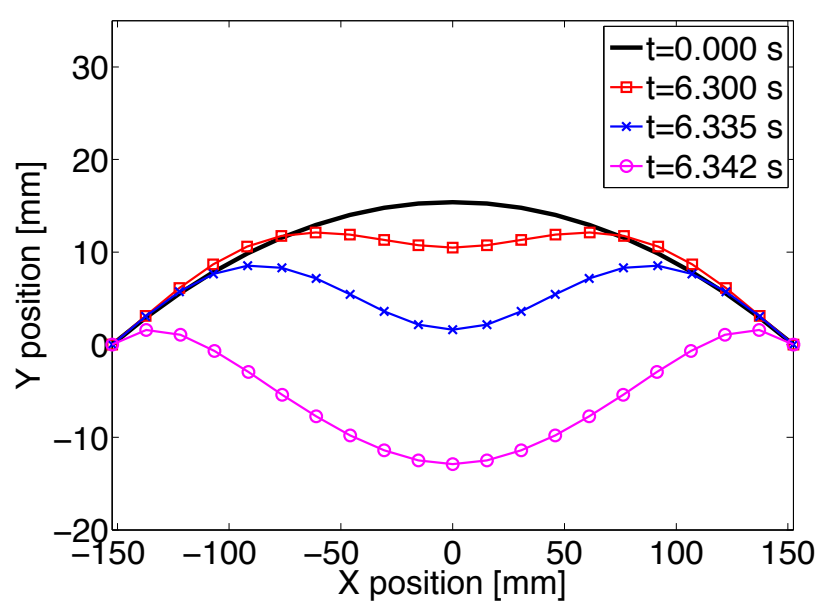

(a) Beam 1

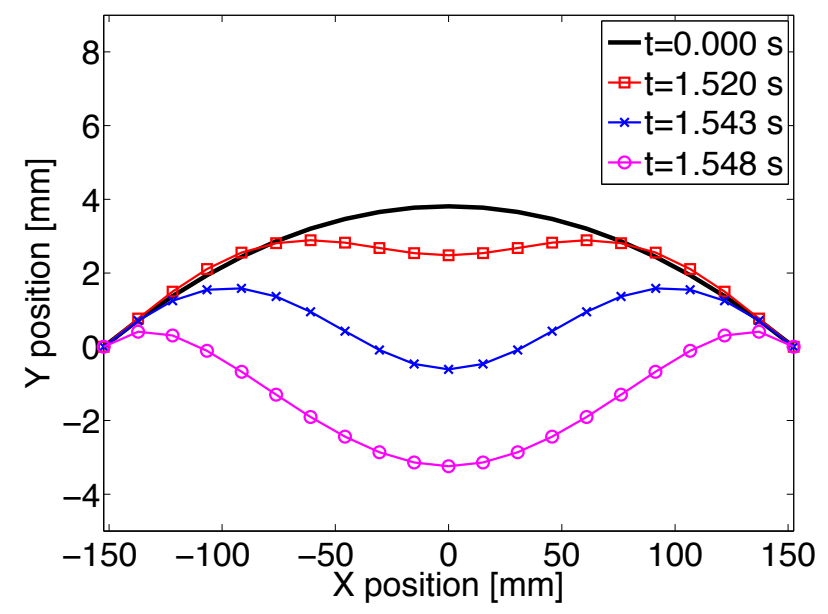

(b) Beam 2

Fig. 7: Arch configurations obtained by the TTBDF scheme at different times.

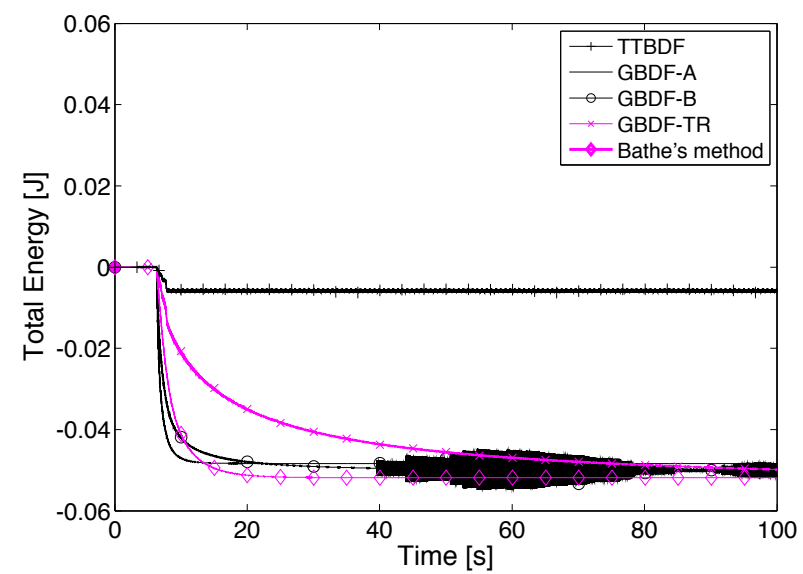

(a) $\Delta t=1 \times 10^{-3} \mathrm{~s}$

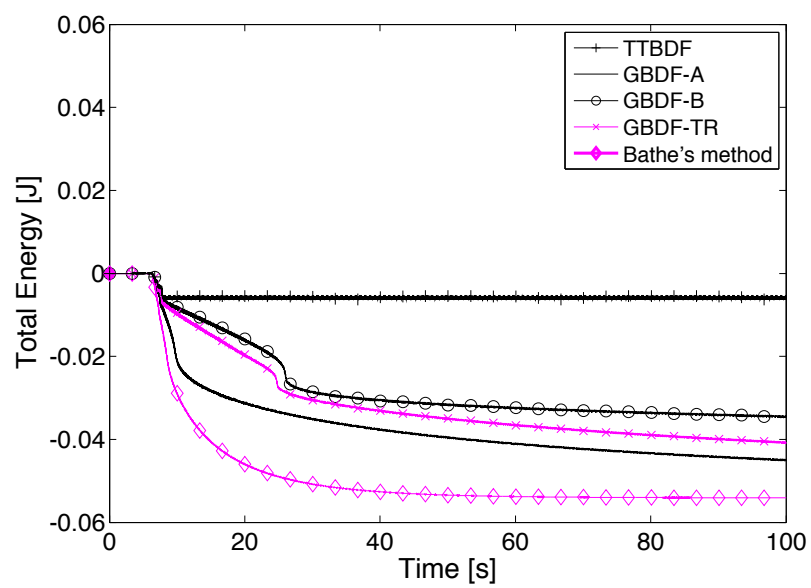

(b) $\delta t=1 / 3 \times 10^{-3} \mathrm{~s}$

Fig. 8: Total energy of Beam 1 obtained using the TTBDF scheme, the GBDF-A, GBDF-B, GBDF-TR, and Bathe's method. 


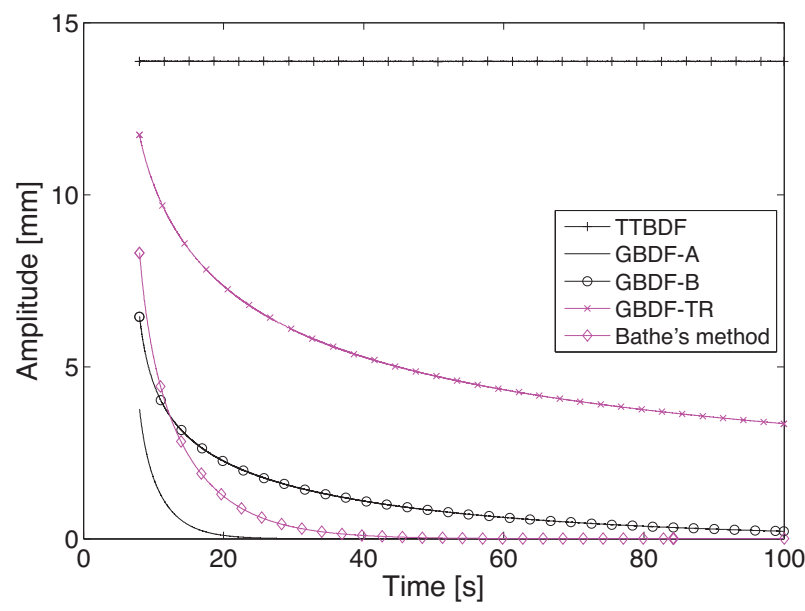

(a) $\Delta t=1 \times 10^{-3} \mathrm{~s}$

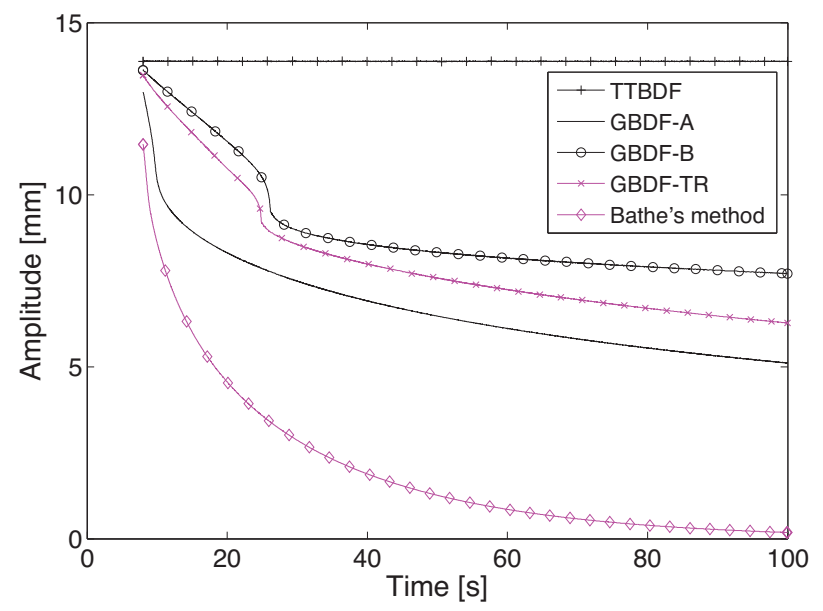

(b) $\delta t=1 / 3 \times 10^{-3} \mathrm{~s}$

Fig. 9: Amplitude decay of Beam 1 obtained using the TTBDF scheme, the GBDF-A, GBDF-B, GBDF-TR, and Bathe's method.

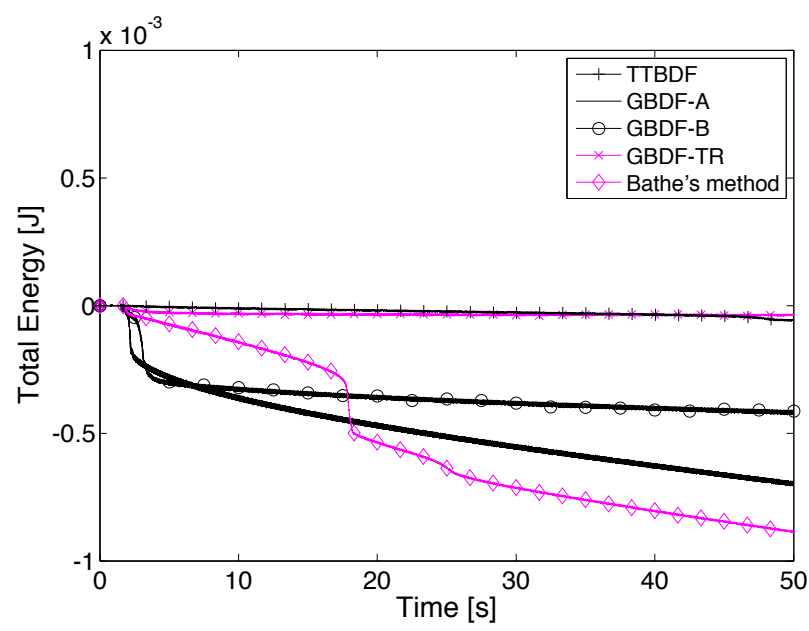

(a) $\Delta t=2 \times 10^{-4} \mathrm{~s}$

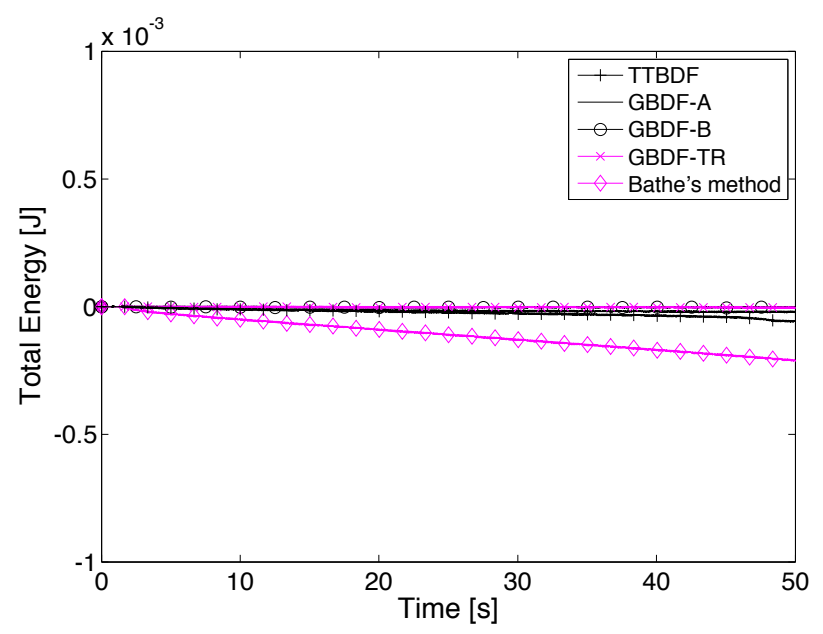

(b) $\delta t=2 / 3 \times 10^{-4} \mathrm{~s}$

Fig. 10: Total energy of Beam 2 obtained using the TTBDF scheme, the GBDF-A, GBDF-B, GBDF-TR, and Bathe's method. 


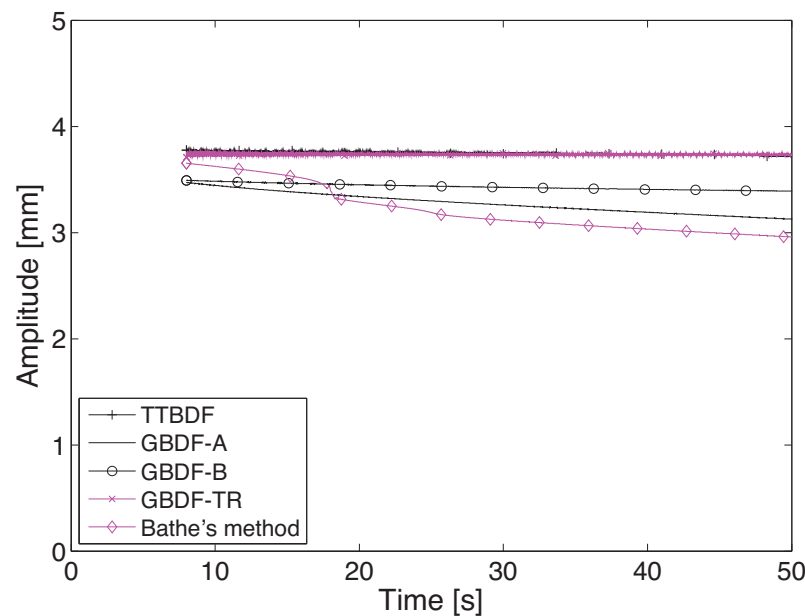

(a) $\Delta t=2 \times 10^{-4} \mathrm{~s}$

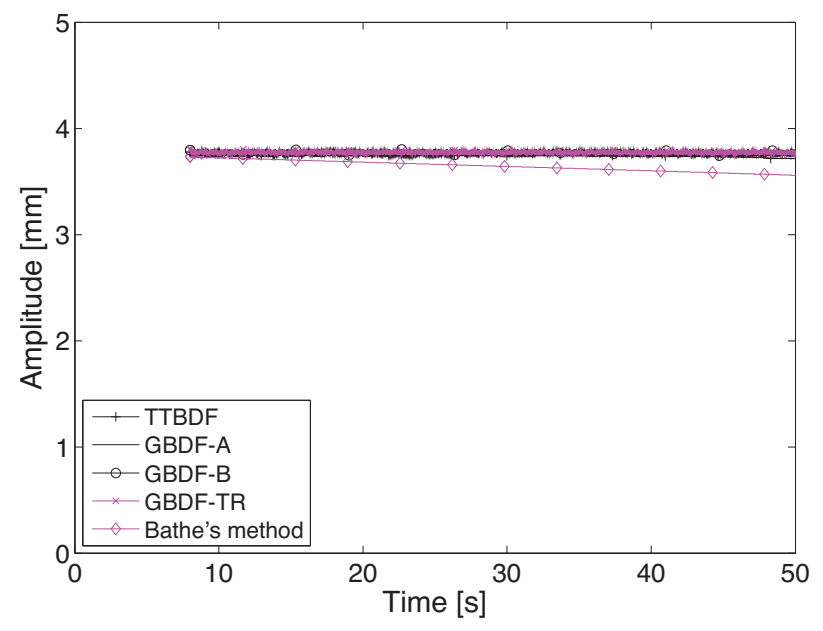

(b) $\delta t=2 / 3 \times 10^{-4} \mathrm{~s}$

Fig. 11: Amplitude decay of Beam 2 obtained using the TTBDF scheme, the GBDF-A, GBDF-B, GBDF-TR, and Bathe's method.

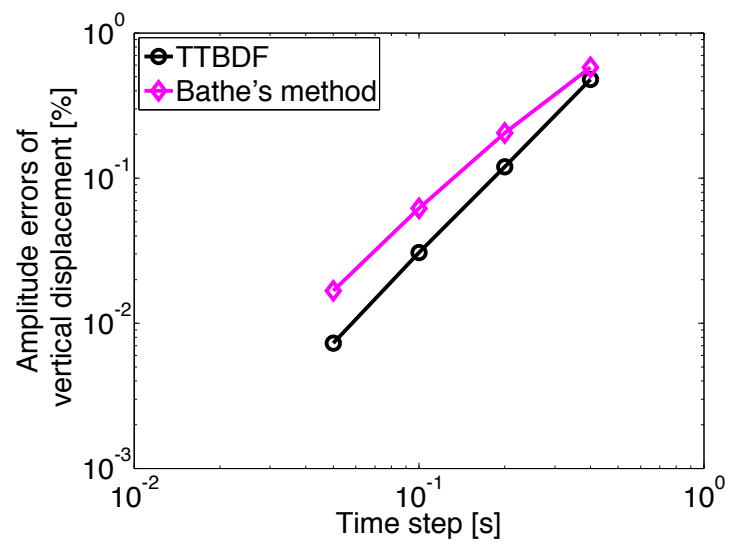

(a) Amplitude errors

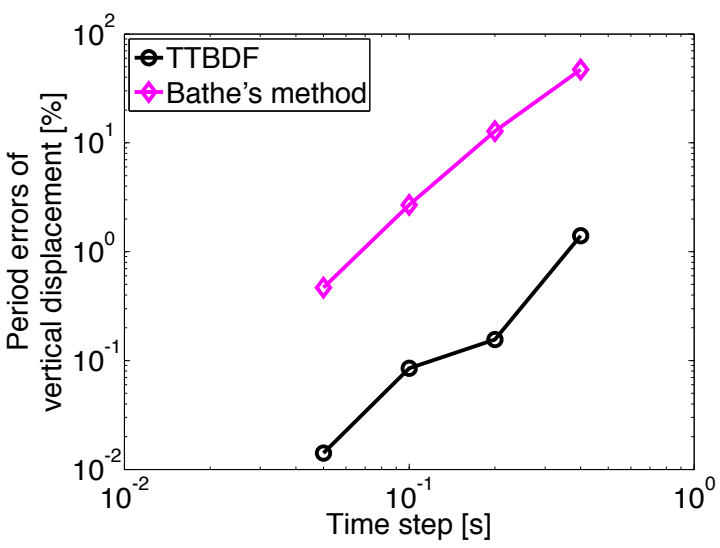

(b) Period errors

Fig. 12: Amplitude and period errors of the vertical displacement of the rigid pendulum. 


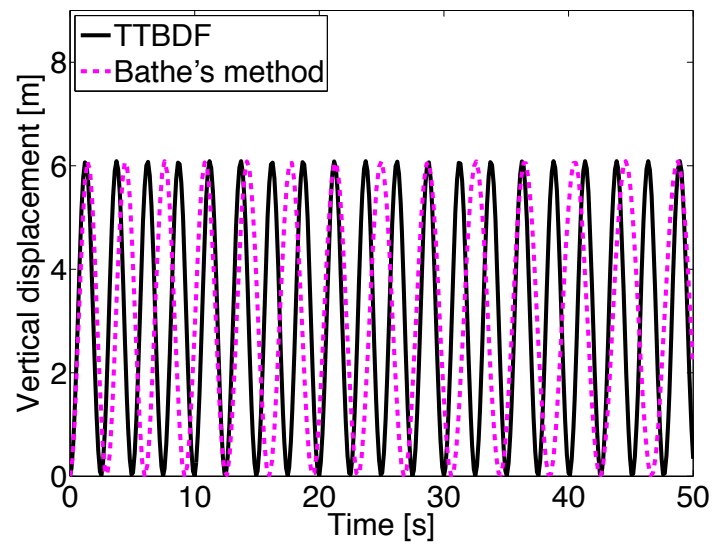

(a) Vertical displacement responses

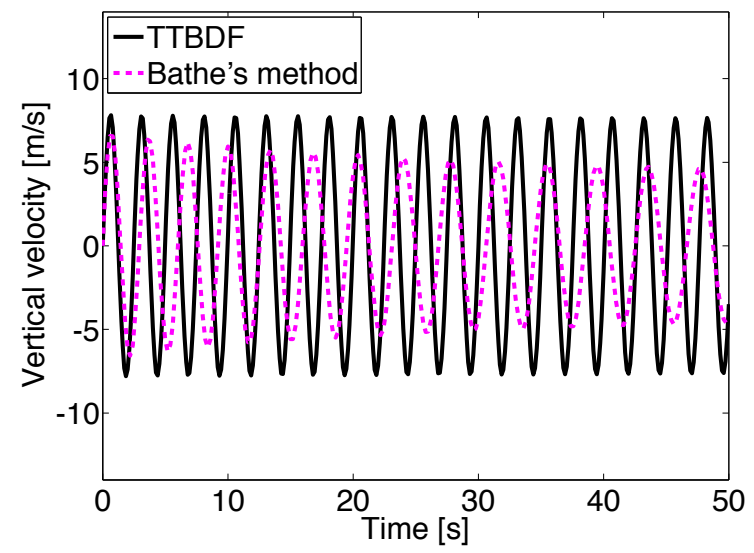

(b) Vertical velocity responses

Fig. 13: Vertical displacement and velocity responses of the rigid pendulum for $\Delta t=0.4 \mathrm{~s}$.

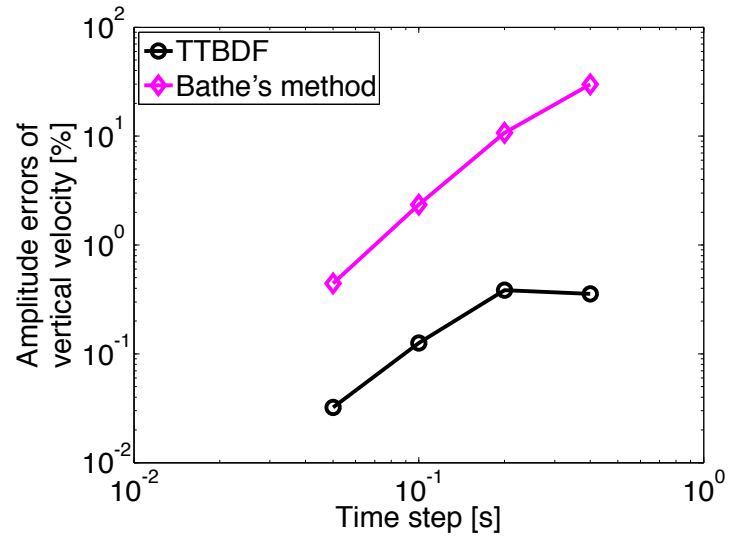

(a) Amplitude errors

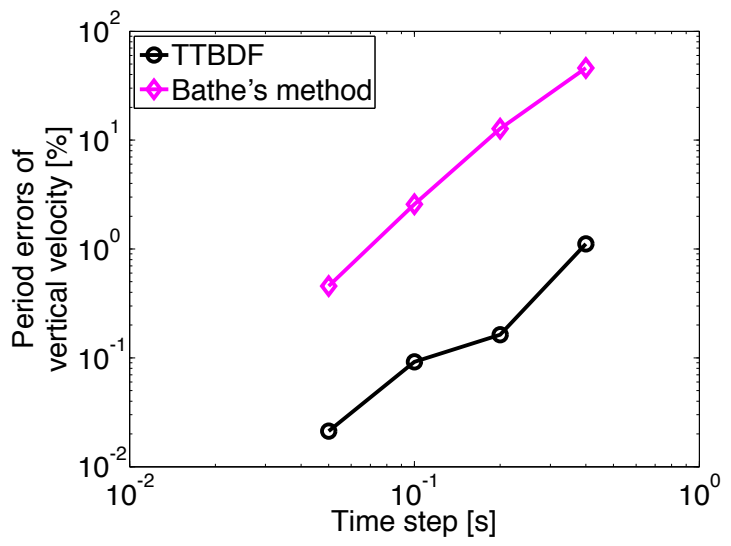

(b) Period errors

Fig. 14: Amplitude and period errors of the vertical velocity of the rigid pendulum.

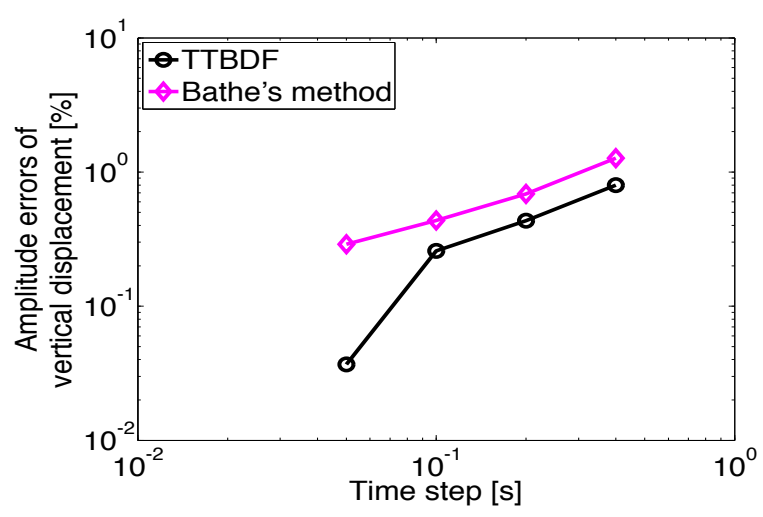

(a) amplitude errors

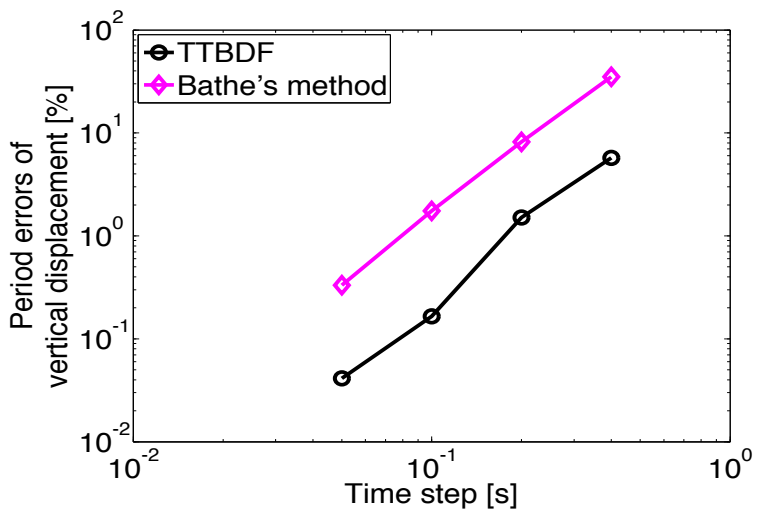

(b) period errors

Fig. 15: Amplitude and period errors of the vertical displacement of the elastic pendulum. 


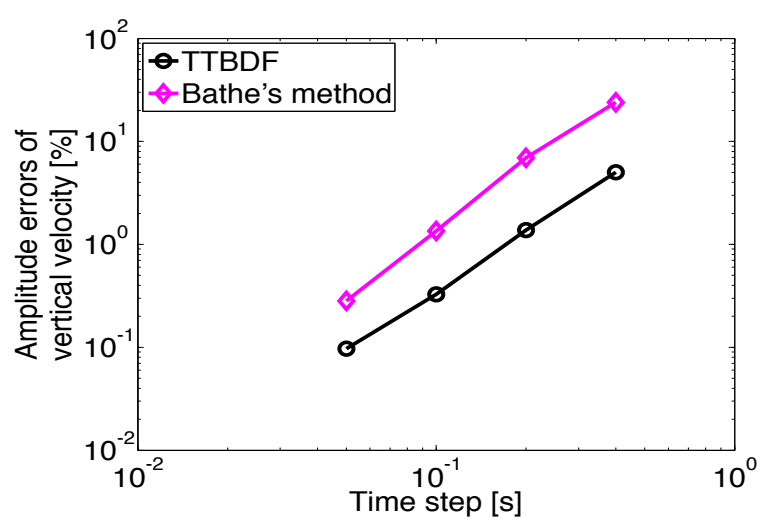

(a) amplitude errors

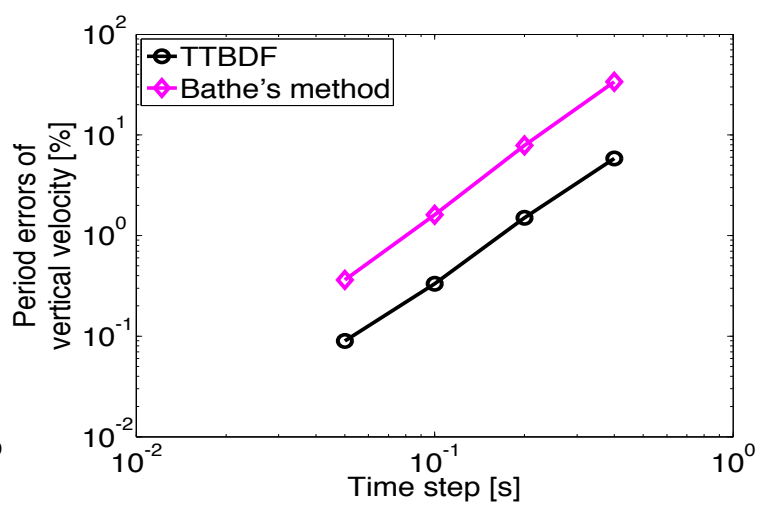

(b) period errors

Fig. 16: Amplitude and period errors of the vertical velocity of the elastic pendulum.

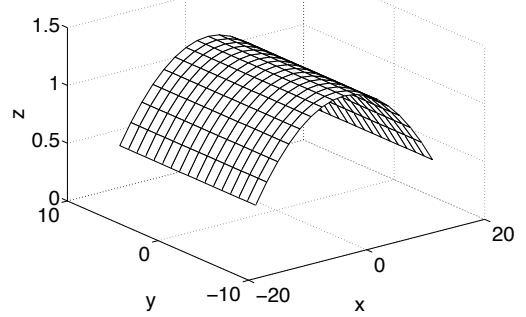

(a) $\mathrm{t}=0$

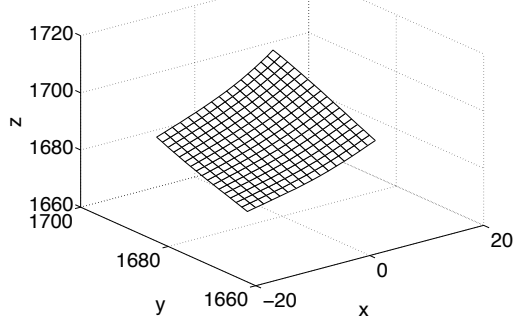

(d) $\mathrm{t}=0.006$

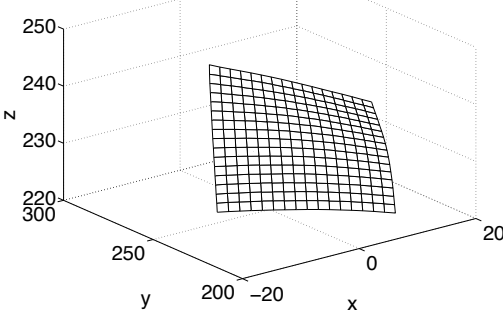

(b) $\mathrm{t}=0.002$

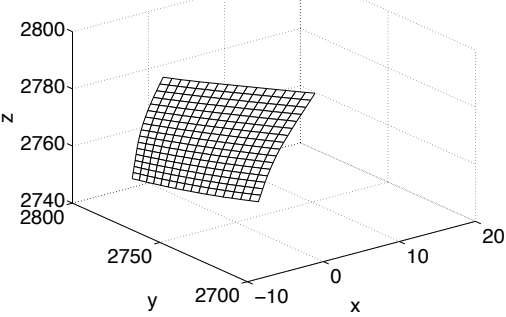

(e) $\mathrm{t}=0.008$

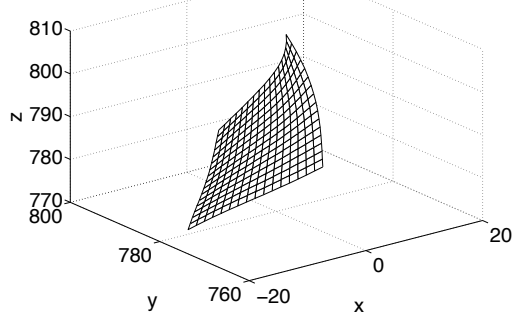

(c) $\mathrm{t}=0.004$

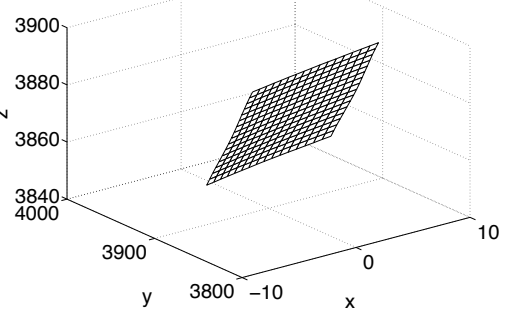

(f) $\mathrm{t}=0.01$

Fig. 19: Snapshots of the cylindrical panel obtained by TTBDF with $\Delta t=2 \times 10^{-4}$. 


\subsubsection{Rigid L-shaped block}

The initial undeformed configuration, dimensions and loading conditions of the L-shaped block are shown in Fig. 20. Two transient traction forces defined by Eq. 15 are applied at the two end faces of the block.

$$
\begin{gathered}
\mathbf{F}_{1}(t)=-\mathbf{F}_{2}(t)=(150,300,450) p(t) \\
p(t)= \begin{cases}t & 0 \leqslant t<2.5 \\
5-t & 2.5 \leqslant t<5 \\
0 & 5 \leqslant t\end{cases}
\end{gathered}
$$

Since the total applied force is zero, the block will simply tumble in space with no net displacement of its center of mass. Like in [23], the units of all model parameters are disregarded (they are assumed to be consistent). Four 27-node continuum elements are used for the spatial discretization of this L-shaped block. The material of the rigid block is described by the NeoHookean law with Young's modulus E $=5 \times 10^{10}$, Poisson's ratio $\nu=0.3$, and density $\rho=1000$.

For each time step, long-time (up to $t=1000)$ simulations are conducted for all methods. A very small time step $\Delta t=0.01$ is used in the initial loading stage $(0 \leqslant t \leqslant 5)$ for all simulations so that the total energy levels of all five algorithms are very close at the end of the loading stage. For a small time step $\Delta t=0.25$, the total energy curves of all algorithms are very similar over the entire simulation time (Fig. 21a). When a much larger time step $\Delta t=1.5$ is used, the GBDF-TR and Bathe's methods become unstable, while the other three methods are still stable and the TTBDF method has the least energy decay (Fig. 21b).

Fig. 22 shows snapshots of the rigid 3D block, which are obtained by the TTBDF method $(\Delta t=1.5)$ at six different time sequences $t=0,9,18,27,36$ and 45 . It can be seen that this rigid L-shaped block has large three-dimensional rotations. The TTBDF method performs well for this problem even at large time steps.

\subsubsection{Deformable L-shaped block}

The deformable block has Young's modulus $\mathrm{E}=5 \times 10^{4}$, Poisson's ratio $\nu=0.3$, and density $\rho=1000$. Similar to the rigid block, a very small time step $\Delta t=0.01$ is used in the initial loading stage to guarantee similar total energy levels at the end of loading stage.

For a small time step $\Delta t=0.25$, the TTBDF method has the least energy decay, closely followed by the GBDFTR method (Fig. 23a). For a larger time step $\Delta t=1.0$, the TTBDF method has significantly less energy dissipation than all other methods (Fig. 23b.
Some snapshots of the deformable 3D block are shown in Fig. 24. These snapshots are obtained by the TTBDF method with $\Delta t=1.0$ at six different time instances $t=0,10,20,30,40$ and 50. One can observe that this deformable L-shaped block has large overall 3D deformations and rotations.

It can be concluded that at large time steps, the TTBDF method not only results in stable solutions but has much less energy dissipation than the three GBDF algorithms and Bathe's method, when simulating the 3D nonlinear dynamic systems involving either large rigid-body motions or elastic displacements. The improved numerical dissipation of the TTBDF method over these composite algorithms is clearly demonstrated.

\section{Conclusions}

Motivated by the numerical difficulties in simulating snap-through buckling problems, we proposed a new composite scheme, the TTBDF method, that employs a similar strategy to that of Bathe's methods [5, $, 3,4$, 6]. The algorithm has three equal sub-steps with the first and second sub-steps using the trapezoidal rule and the last sub-step using a three-point BDF-like scheme 23 . The proposed method significantly improves accuracy and computational efficiency in simulating structures undergoing snap-through when compared to other methods with similar approach, as shown through the examples in this paper.

The performance of the proposed method on free moving shells and rigid and deformable L-shaped blocks and on pendulum problems was also tested. The examples presented show the reliability and effectiveness of the TTBDF method in solving large deformation, large displacement and rotation problems. Comparison with other schemes from the BDF class and composite schemes that have a member of BDF class as a substep show that TTBDF method is numerically stable and often more efficient.

Acknowledgements The work has been funded in part by AFOSR under the grant no. FA9550-09-1-0201 and by DOD under the High Performance Computing Modernization Program (HPCMP) under the grant GS04T09DBC0017. Their support is greatly appreciated. Support was also received from the Data Analysis and Visualization Cyberinfrastructure funded by NSF under grant OCI-0959097 and the Shared University Grid at Rice funded by NSF under Grant EIA0216467, and a partnership between Rice University, Sun Microsystems, and Sigma Solutions, Inc. 


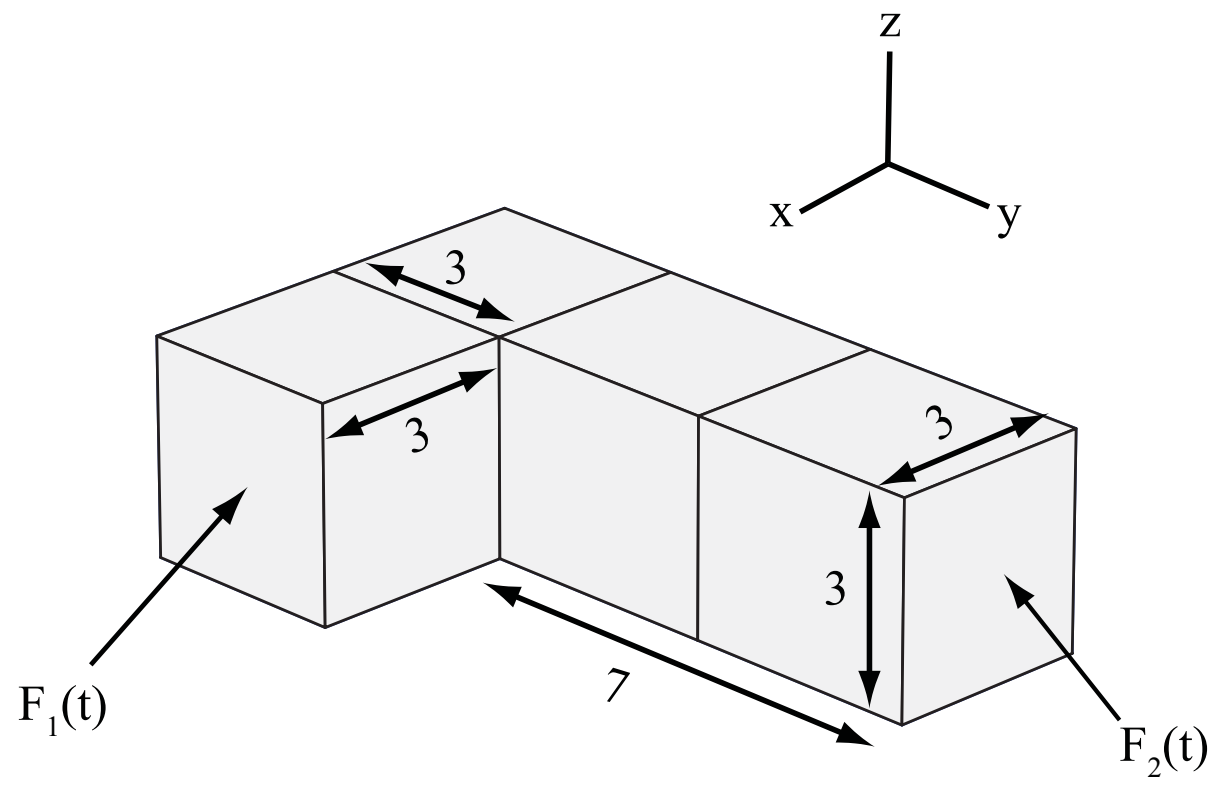

Fig. 20: Three-dimensional L-shaped Block

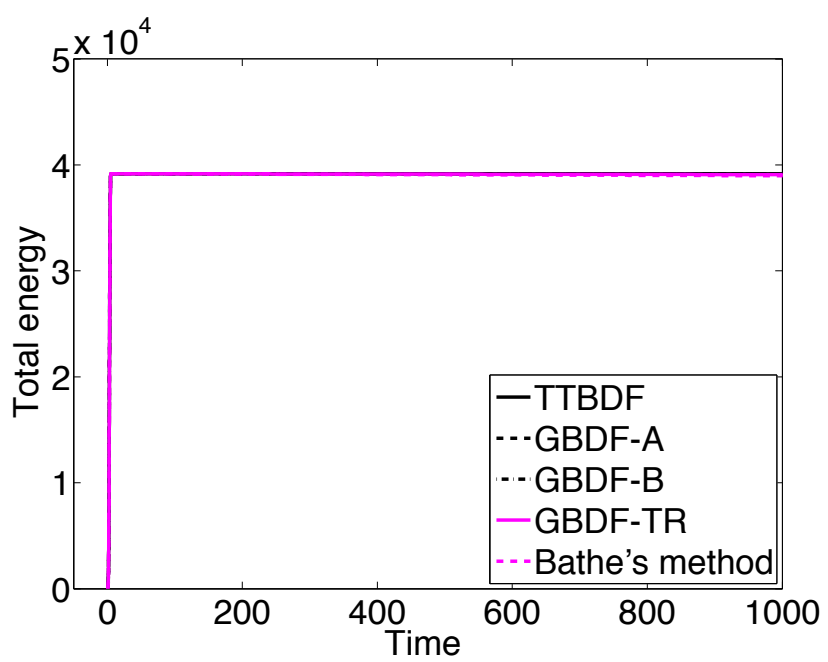

(a) $\Delta t=0.25$

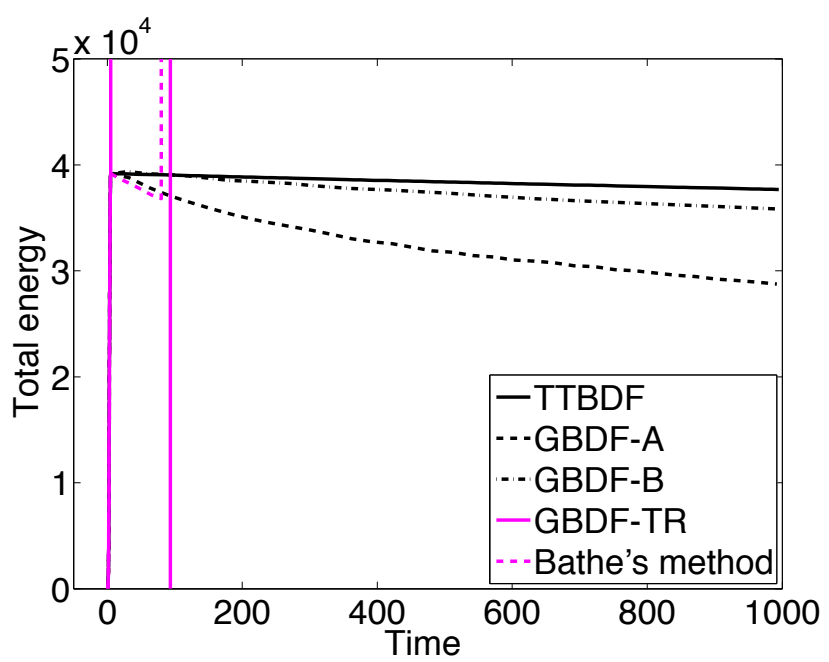

(b) $\Delta t=1.5$

Fig. 21: Total energy of the rigid L-shaped block.

\section{References}

1. Armero, F., Romero, I.: On the formulation of highfrequency dissipative time-stepping algorithms for nonlinear dynamics. part i: low-order methods for two model problems and nonlinear elastodynamics. Computer Methods in Applied Mechanics and Engineering 190(20), 2603-2649 (2001)

2. Armero, F., Romero, I.: On the formulation of highfrequency dissipative time-stepping algorithms for nonlinear dynamics. part ii: second-order methods. Computer Methods in Applied Mechanics and Engineering 190(51), 6783-6824 (2001)
3. Baig, M.M.I., Bathe, K.J.: On direct time integration in large deformation dynamic analysis. In: 3rd M.I.T. Conference on Computational Fluid and Solid Mechanics, pp. 1044-1047 (2005)

4. Bathe, K.J.: Conserving energy and momentum in nonlinear dynamics: A simple implicit time integration scheme. Computers and Structures 85(7-8), 437-445 (2007)

5. Bathe, K.J., Baig, M.M.I.: On a composite implicit time integration procedure for nonlinear dynamics. Computers and Structures 83(31-32), 2513-2524 (2005)

6. Bathe, K.J.r., Noh, G.: Insight into an implicit time integration scheme for structural dynamics. Computers and 


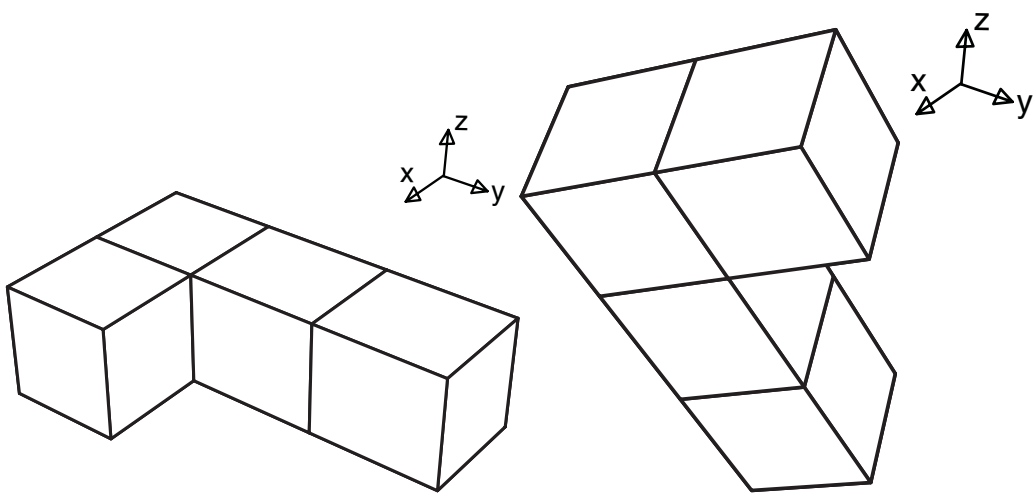

(a) $t=0$

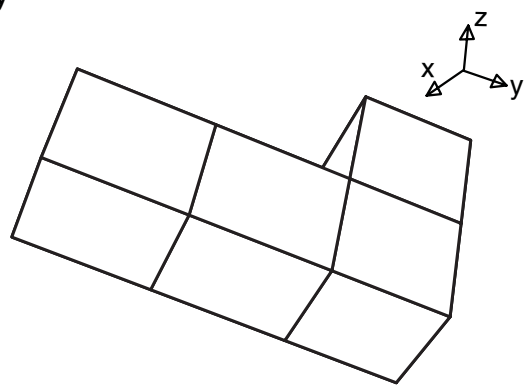

(c) $\mathrm{t}=18$

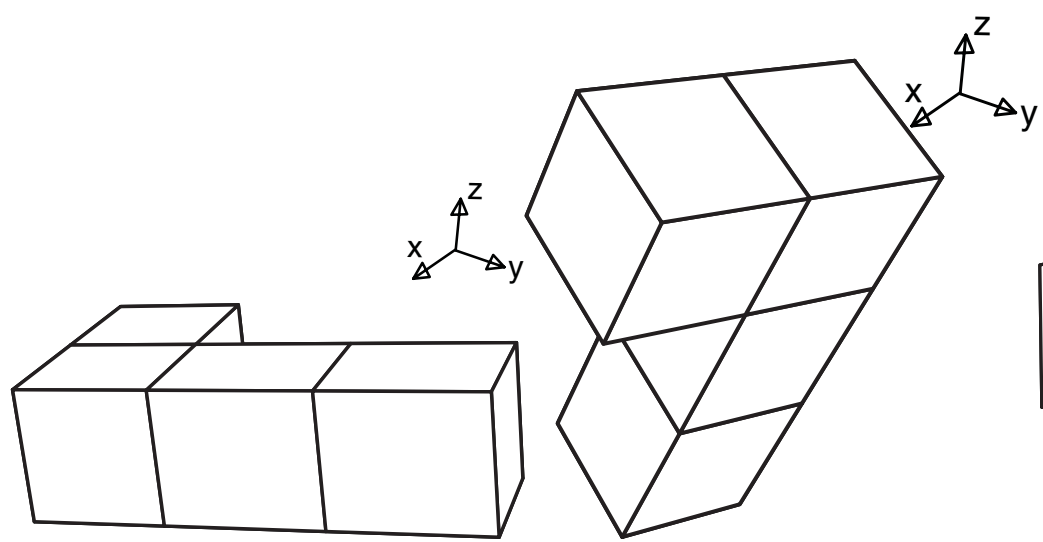

(d) $\mathrm{t}=27$

(e) $\mathrm{t}=36$

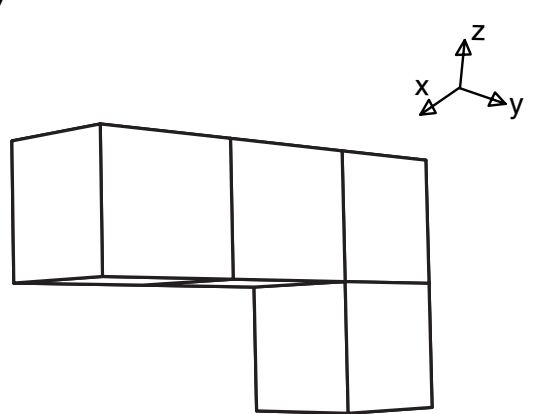

(f) $\mathrm{t}=45$

Fig. 22: Snapshots of the rigid L-shaped block obtained by TTBDF with $\Delta t=1.5$.

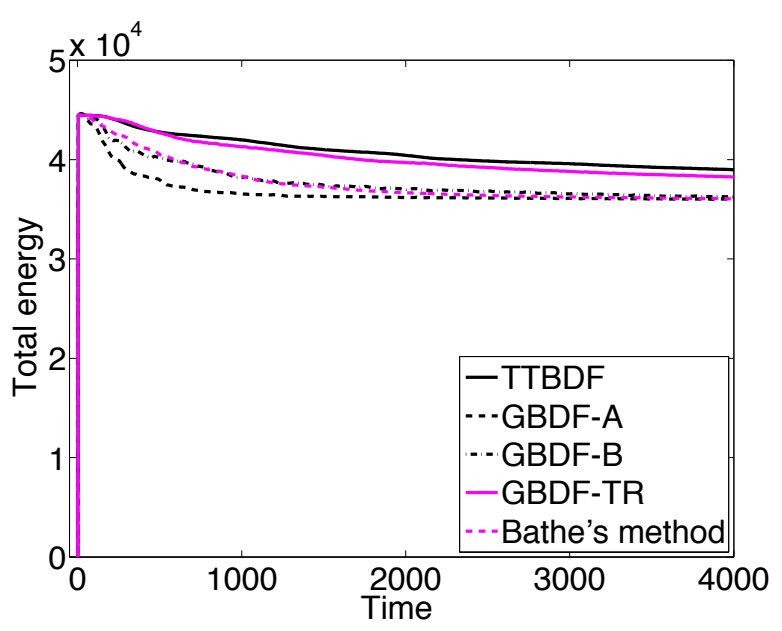

(a) $\Delta t=0.25$

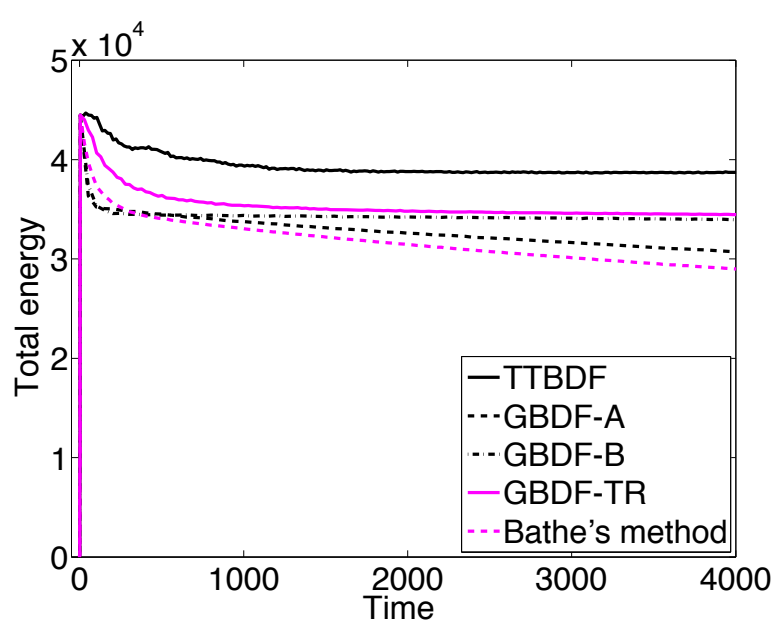

(b) $\Delta t=1.0$

Fig. 23: Total energy of the deformable L-shaped block.

Structures 98-99(C), 1-6 (2012)

7. Bauchau, O., Damilano, G., Theron, N.J.: Numerical integration of non-linear elastic multi-body systems. International Journal for Numerical Methods in Engineering
38(16), 2727-2751 (1995)

8. Bauchau, O., Theron, N.: Energy decaying scheme for non-linear beam models. Computer Methods in Applied Mechanics and Engineering 134(1), 37-56 (1996) 


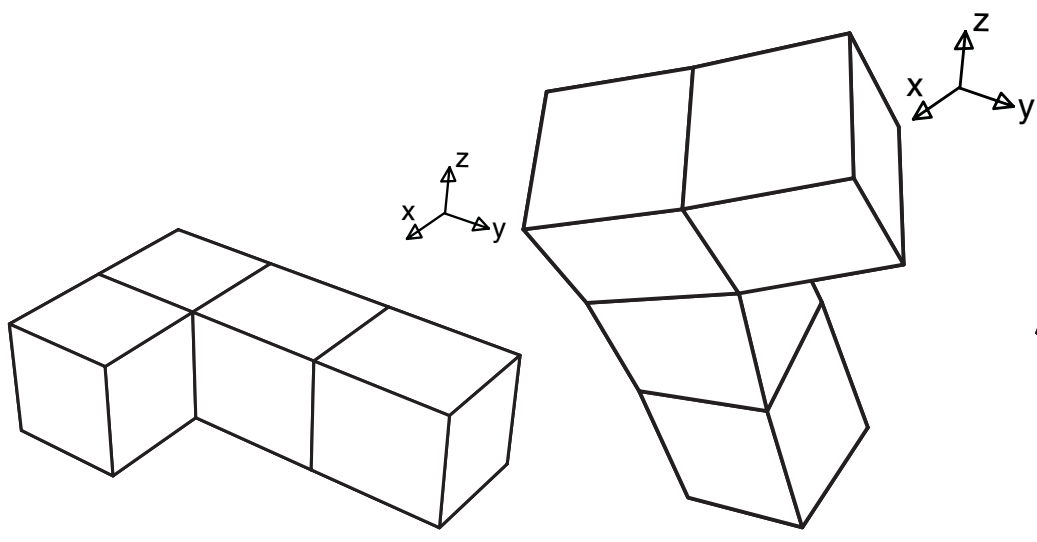

(a) $t=0$ (b) $t=10$

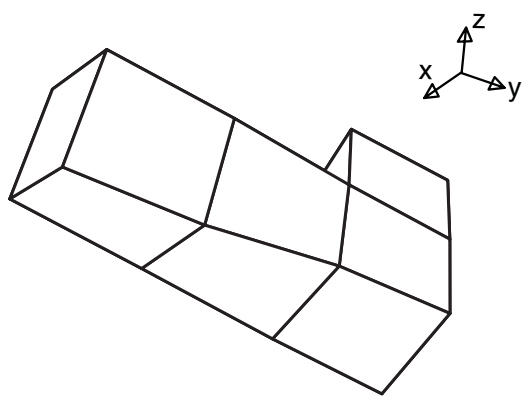

(c) $\mathrm{t}=20$

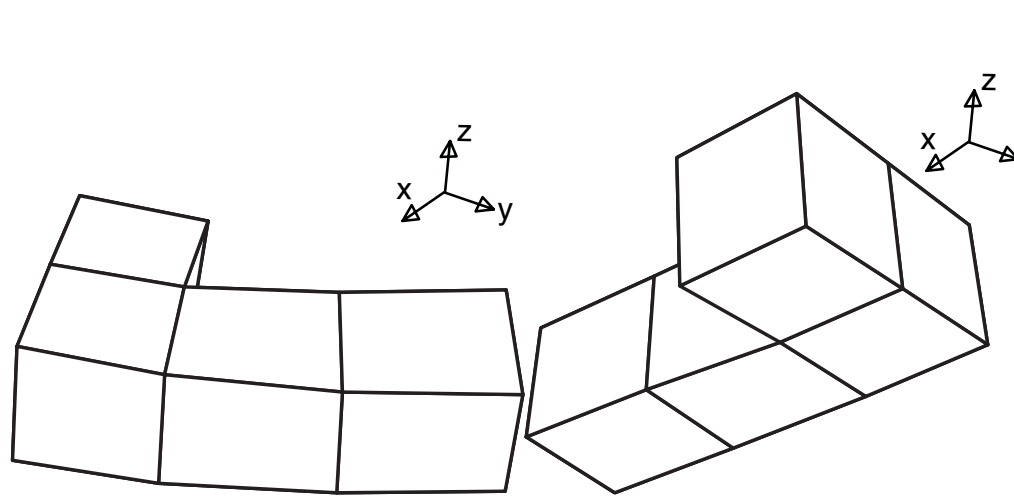

(d) $\mathrm{t}=30$

(e) $\mathrm{t}=40$

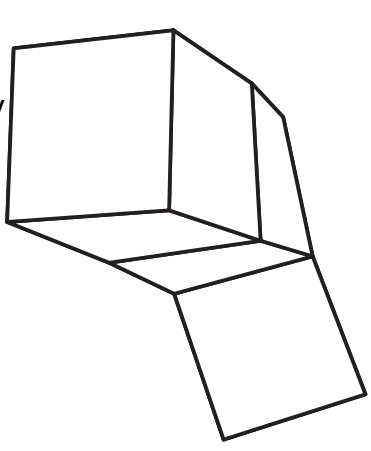

(f) $\mathrm{t}=50$<smiles></smiles>

Fig. 24: Snapshots of the deformable L-shaped block obtained by TTBDF with $\Delta t=1.0$.

9. Bauchau, O., Theron, N.: Energy decaying scheme for nonlinear elastic multi-body systems. Computers \& Structures 59(2), 317-331 (1996)

10. Bauchau, O.A., Bottasso, C.L.: On the design of energy preserving and decaying schemes for flexible, nonlinear multi-body systems. Computer Methods in Applied Mechanics and Engineering 169(1), 61-79 (1999)

11. Borri, M., Bottasso, C.L., Trainelli, L.: Integration of elastic multibody systems by invariant conserving/dissipating algorithms. i. formulation. Computer methods in applied mechanics and engineering 190(29), 3669-3699 (2001)

12. Bottasso, C.L., Borri, M.: Energy preserving/decaying schemes for non-linear beam dynamics using the helicoidal approximation. Computer Methods in Applied Mechanics and Engineering 143(3), 393-415 (1997)

13. Bottasso, C.L., Borri, M.: Integrating finite rotations. Computer Methods in Applied Mechanics and Engineering 164(3), 307-331 (1998)

14. Bottasso, C.L., Borri, M., Trainelli, L.: Integration of elastic multibody systems by invariant conserving/dissipating algorithms. ii. numerical schemes and applications. Computer Methods in Applied Mechanics and Engineering 190(29), 3701-3733 (2001)

15. Brank, B., Briseghella, L., Tonello, N., Damjanic, F.B.: On non-linear dynamics of shells: implementation of energy-momentum conserving algorithm for a finite ro- tation shell model. International journal for numerical methods in engineering 42(3), 409-442 (1998)

16. Campello, E., Pimenta, P., Wriggers, P.: An exact conserving algorithm for nonlinear dynamics with rotational dofs and general hyperelasticity. part 2: shells. Computational Mechanics 48(2), 195-211 (2011)

17. Chandra, Y.: Snap through of curved beam. Master's thesis, University of Illinois at Urbana-Champaign (2009)

18. Chandra, Y.: Transient behavior of curved structures. Ph.D. thesis, Rice University (2013)

19. Chandra, Y., Stanciulescu, I., Eason, T., Spottswood, M.: Numerical pathologies in snap-through simulations. Engineering Structures 34, 495-504 (2012)

20. Chandra, Y., Stanciulescu, I., Virgin, L.N., Eason, T.G., Spottswood, S.M.: A numerical investigation of snapthrough in a shallow arch-like model. Journal of Sound and Vibration 332(1), 2532-2548 (2013)

21. Chandra, Y., Wiebe, R., Stanciulescu, I., Virgin, L., Spottswood, S., Eason, T.: Characterizing dynamic transitions associated with snap-through of clamped shallow arches. Journal of Sound and Vibration (2013)

22. Chung, J., Hulbert, G.: Time integration algorithm for structural dynamics with improved numerical dissipation: the generalized- $\alpha$ method. Journal of Applied Mechanics, Transactions ASME 60(2), 371-375 (1993)

23. Dong, S.: BDF-like methods for nonlinear dynamic analysis. Journal of Computational Physics 229(8), 3019-3045 (2010) 
24. Gonzalez, O.: Exact energy and momentum conserving algorithms for general models in nonlinear elasticity. Computer Methods in Applied Mechanics and Engineering 190(13), 1763-1783 (2000)

25. Hilber, H., Hughes, T., Taylor, R.: Improved Numerical Dissipation for Time Integration Algorithms in Structural Dynamics. Earthquake Engineering and Structural Dynamics 5(3), 283-292 (1977)

26. Hughes, T.J.R.: The Finite Element Method: Linear Static and Dynamic Finite Element Analysis. PrenticeHall, Englewood Cliffs, New Jersey (1987)

27. Ibrahimbegović, A., Mamouri, S.: Energy conserving/decaying implicit time-stepping scheme for nonlinear dynamics of three-dimensional beams undergoing finite rotations. Computer Methods in Applied Mechanics and Engineering 191(37-38), 4241-4258 (2002)

28. Ibrahimbegović, A., Mamouri, S., Taylor, R.L., Chen, A.J.: Finite element method in dynamics of flexible multibody systems: modeling of holonomic constraints and energy conserving integration schemes. Multibody System Dynamics 4(2-3), 195-223 (2000)

29. Kalmár-Nagy, T., Stanciulescu, I.: Can complex systems really be simulated? Applied Mathematics and Computation 227, 199-211 (2014)

30. Kuhl, D., Crisfield, M.: Energy-conserving and decaying algorithms in non-linear structural dynamics. International Journal for Numerical Methods in Engineering 45(5), 569-599 (1999)

31. Kuhl, D., Ramm, E.: Constraint energy momentum algorithm and its application to non-linear dynamics of shells. Computer Methods in Applied Mechanics and Engineering 136(3), 293-315 (1996)

32. Kuhl, D., Ramm, E.: Generalized energy-momentum method for non-linear adaptive shell dynamics. Computer Methods in Applied Mechanics and Engineering 178(3), 343-366 (1999)

33. Laursen, T., Meng, X.: A new solution procedure for application of energy-conserving algorithms to general constitutive models in nonlinear elastodynamics. Computer Methods in Applied Mechanics and Engineering 190(46), 6309-6322 (2001)

34. Newmark, N.M.: A method of computation for structural dynamics. Journal of the Engineering Mechanics Division 85, 67-94 (1959)

35. Pimenta, P., Campello, E., Wriggers, P.: An exact conserving algorithm for nonlinear dynamics with rotational dofs and general hyperelasticity. part 1: Rods. Computational Mechanics 42(5), 715-732 (2008)

36. Romero, I., Armero, F.: Numerical integration of the stiff dynamics of geometrically exact shells: an energydissipative momentum-conserving scheme. International journal for numerical methods in engineering 54(7), 1043-1086 (2002)

37. Sansour, C., Wagner, W., Wriggers, P., Sansour, J.: An energy-momentum integration scheme and enhanced strain finite elements for the non-linear dynamics of shells. International Journal of Non-Linear Mechanics 37(4), 951-966 (2002)

38. Sansour, C., Wriggers, P., Sansour, J.: Nonlinear dynamics of shells: theory, finite element formulation, and integration schemes. Nonlinear Dynamics 13(3), 279-305 (1997)

39. Sansour, C., Wriggers, P., Sansour, J.: On the design of energy-momentum integration schemes for arbitrary continuum formulations. applications to classical and chaotic motion of shells. International journal for numerical methods in engineering 60(15), 2419-2440 (2004)
40. Simo, J., Tarnow, N.: The discrete energy-momentum method. Conserving algorithms for nonlinear elastodynamics. ZAMP Zeitschrift für angewandte Mathematik und Physik 43(5), 757-792 (1992)

41. Simo, J., Tarnow, N.: A new energy and momentum conserving algorithm for the non-linear dynamics of shells. International Journal for Numerical Methods in Engineering 37(15), 2527-2549 (1994)

42. Simo, J., Tarnow, N., Doblare, M.: Non-linear dynamics of three-dimensional rods: Exact energy and momentum conserving algorithms. International Journal for Numerical Methods in Engineering 38(9), 1431-1473 (1995)

43. Taylor, R.L.: FEAP - finite element analysis program (2014). URL http://www.ce.berkeley/feap

44. Virgin, L.N.: Vibration of Axially-Loaded Structures. Cambridge University Press (2007)

45. Wiebe, R., Stanciulescu, I.: Inconsistent stability of Newmark's method in structural dynamics applications. Journal of ... (2014)

46. Wiebe, R., Virgin, L., Stanciulescu, I., Spottswood, S., Eason, T.: Characterizing Dynamic Transitions Associated With Snap-Through: A Discrete System. Journal of Computational and Nonlinear Dynamics 8, 011,010 (2013)

47. Wood, W., Bossak, M., Zienkiewicz, O.: An alpha modification of newmark's method. International Journal for Numerical Methods in Engineering 15(10), 1562-1566 (1980)

48. Zhong, H., Crisfield, M.: An energy-conserving corotational procedure for the dynamics of shell structures. Engineering Computations 15(5), 552-576 (1998) 